\title{
Studies of Electrolytic Conductivity of Some Polyelectrolyte Solutions: Importance of the Dielectric Friction Effect at High Dilution
}

\author{
Anis Ghazouani, Sondes Boughammoura, and Jalel M'Halla \\ Faculty of Sciences of Monastir, UR "Electrolytes”, University of Monastir, 5000 Monastir, Tunisia \\ Correspondence should be addressed to Jalel M’Halla; jalel.mhalla@fsm.rnu.tn
}

Received 27 June 2012; Accepted 29 August 2012

Academic Editor: Jean-François Dufrêche

Copyright (C) 2013 Anis Ghazouani et al. This is an open access article distributed under the Creative Commons Attribution License, which permits unrestricted use, distribution, and reproduction in any medium, provided the original work is properly cited.

\begin{abstract}
We present a general description of conductivity behavior of highly charged strong polyelectrolytes in dilute aqueous solutions taking into account the translational dielectric friction on the moving polyions modeled as chains of charged spheres successively bounded and surrounded by solvent molecules. A general formal limiting expression of the equivalent conductivity of these polyelectrolytes is presented in order to distinguish between two concentration regimes and to evaluate the relative interdependence between the ionic condensation effect and the dielectric friction effect, in the range of very dilute solutions for which the stretched conformation is favored. This approach is illustrated by the limiting behaviors of three polyelectrolytes (sodium heparinate, sodium chondroitin sulfate, and sodium polystyrene sulphonate) characterized by different chain lengths and by different discontinuous charge distributions.
\end{abstract}

\section{Introduction}

Conductivity is a powerful technique of high accuracy allowing the qualitative and the quantitative detection of ionic species even at low concentrations. On the other hand, it is well known that for electrolytes (or for electrolytes mixtures), it exists a universal linear limiting law relating the equivalent conductivity at high dilution to the square root of ionic strength $I^{1 / 2}$. This concentration effect is caused by two sorts of ionic frictions: the electrophoretic effect and the ionic relaxation effect. This limiting law has been extended in the case of semidilute simple electrolytes [1-4]. In contrast, a completely satisfactory theory to describe the dynamic behavior in general and particularly the electrolytic conductivity of dilute flexible polyelectrolytes in aqueous solution is not yet available despite some interesting progress toward this objective [5-16]. This difficulty arises from the complex interdependence between polyion conformation, ionic condensation, screening effect, and frictional forces. Moreover, it is important to underline that these different attempts have ignored the influence of the translational dielectric friction on moving polyions as well as its dependence on concentration $[17,18]$. The present paper is a supplementary contribution toward this goal in order to propose a general formal limiting equation expressing the influence of the effects cited above on the equivalent conductivity of polyelectrolytes, and applicable in the range of very dilute solutions for which the stretched conformation is favored and for which the electrophoretic effects and ionic relaxation effects are negligible. This approach will be illustrated by the behaviors of three polyelectrolytes (sodium heparinate, sodium chondroitin sulfate, and sodium polystyrene sulphonate) characterized by different chain lengths and by different discontinuous charge distributions.

\section{Theoretical Model and Parameters}

2.1. Presentation of the Cylindrical Model. For dilute polyelectrolyte solutions the long chains of ionized polymers are generally assumed to be completely stretched [5-13], so that each chain can be modeled as a cylinder of $R_{C}$ radius, $L_{S}$ structural length $\left(L_{S}=A B\right)$ and $Z_{S} e$ structural charge. On 
the other hand, as the linear distribution of the total charge $Z_{S} e$ is uniform, then the distance of separation between two successive ionizable groups is $b_{S}=L_{S} /\left|Z_{S}\right|$. Because of the ionic condensation effect, these charged groups are, in absence of additional salt (salt-free), partially neutralized only by counterions " $i$ " of $Z_{i} e$ charge and $R_{i}$ effective radius. According to Manning's rod-like model $\left(R_{C} / L_{S} \rightarrow 0\right)$ [612], the degree of ionic condensation $\left(1-\alpha_{M}\right)$ is independent on the counterions concentration $C_{i}$ so that $\alpha_{M}=b_{S} /\left|Z_{i}\right| L_{B}$, where $L_{B}=e^{2} / \varepsilon_{o} k_{B} T$ is the Bjerrum length, $k_{B}$ is the Boltzmann constant, and $\varepsilon_{o}$ is the permittivity of the solvent. In fact, experimental conductivity results are not in general in conformity with the current polyelectrolyte theories $[10,11$, $14,15]$. In particular, we have proved that for more realistic polyelectrolyte models (rod-like or cylindrical models with finite length and models having ellipsoidal conformations of $A, B$ focuses with $A B=L<L_{S}$ ) [9-11], the corresponding degree of dissociation $\alpha$ obeys in general to the Ostwald's principle of dilution and consequently $\alpha \rightarrow 1$ when $C_{i} \rightarrow 0$. The degree $\alpha$ was calculated on the basis of the two-state model [10] (double layer) proposed by Sélégny, Manning, Dobrynin, and Rubinstein [6-8], but in a different way. According to this approach, the condensed counterions are distributed on an ellipsoidal first layer of $A, B$ focuses and $R$ minor axis, whereas the free counterions constitute the ionic atmosphere which can be represented by an ellipsoidal second layer having the same focuses $A, B$ and a $d_{P}$ minor axis (see Figure 1). The thickness $d_{P}$ is a function of $R, \alpha$ and $C_{i}$ via the Debye-MSA screen parameter $\Gamma_{P M S A}[1]$ and the Debye length $\chi_{D}^{-1}$. Notice that both first and second layers are equipotential surfaces of total charges equal, respectively, to $-(1-\alpha) Z_{S} e$ and $-\alpha Z_{S} e$. In the case of the cylindrical model $\left(A B=L_{S}, R \cong R_{C}\right.$ and $\left.\alpha \equiv \alpha_{C}\right)$, calculation of $\alpha_{C}$ needs the resolution of the following ensemble of implicit equations:

$$
\begin{aligned}
\frac{\left(1-\alpha_{C}\right)}{\alpha_{C}}= & \left(1-V_{P} C_{P}\right)^{-1} V_{P} C_{P} \\
& \times\left[\frac{g\left(R_{C}, L_{S}\right)}{g\left(d_{P}, L_{S}\right)}\right]^{\left\{\left(\alpha_{C}+\left|Z_{i} / Z_{S}\right|\right) / \alpha_{M}\right\}}, \\
g\left(x, L_{S}\right)= & \frac{\left[\left(4 x^{2}+L_{S}^{2}\right)^{1 / 2}+L_{S}\right]}{\left[\left(4 x^{2}+L_{S}^{2}\right)^{1 / 2}-L_{S}\right]} \\
d_{P}= & R_{C}+\frac{1}{2 \Gamma_{P M S A}}, \\
2 \Gamma_{P \mathrm{MSA}}= & \frac{\left[-1+\left(1+4 \chi_{D} R_{i}\right)^{1 / 2}\right]}{2 R_{i}} ; \\
\chi_{D}= & \left(4 \pi L_{B} Z_{i}^{2} \alpha_{C} C_{i}\right)^{1 / 2} .
\end{aligned}
$$

$C_{i}$ and $C_{P}$ are the microscopic concentrations (number of particles $/ \AA^{3}$ ) of, respectively, the counterions and the polyions. $V_{P}=\pi R_{C}^{2} L_{S}$ is the structural volume of the cylindrical polyion. $g\left(R_{C}, L_{S}\right)$ and $g\left(d_{P}, L_{S}\right)$ are "the configuration functions" which depend on the conformation of the polyion.

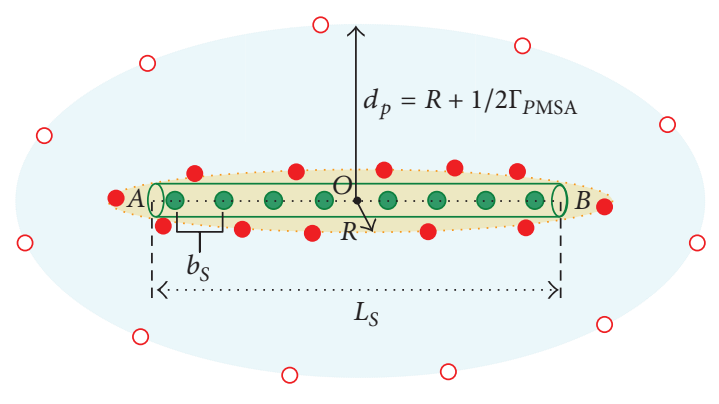

Condensed counterion

Free counterion

Figure 1: Representation of a polyelectrolyte according to the cylindrical model.

Notice first that (1) is a generalization to cylindrical polyions, of the Fuoss expression relative to the ionic association of simple electrolytes; and second, that in the restrictive case of Manning's model $\left(R_{C} / L_{S} \rightarrow 0\right)$ and for dilute solutions $\left(R_{C} / d_{P} \ll 1\right), \alpha_{C}$ increases very slowly with dilution so that $\alpha_{C}$ remains approximately constant in a large range of low concentrations. However, $\alpha_{C}$ approaches toward $\alpha_{M}$ only for some particular polyelectrolytes [10].

Notice also that (1) can be applied for ellipsoidal polyions of any shape (i.e., $L \neq L_{S}, R \neq R_{C}$ and for all $V_{P}$ ).

\subsection{Theoretical Conductivity of Cylindrical Polyelectrolytes} in Dilute Solutions. In practice we measure the specific conductance $\chi$ of the polyelectrolyte solution in $\mathrm{S} \cdot \mathrm{cm}^{-1} \cdot \chi$ is related to the equivalent ionic conductivities $\lambda_{P}$ and $\lambda_{i}$, respectively, of the polyion and the counterion as follows:

$$
\chi=10^{-3}\left[\alpha_{C}\left|Z_{S}\right| \lambda_{P} \mathbf{C}_{P}^{\circ}+\alpha_{C}\left|Z_{i}\right| \lambda_{i} \mathbf{C}_{i}^{\circ}\right]
$$

where $\mathbf{C}_{P}^{\circ}$ is the molar concentration of the polyions and $\mathbf{C}_{i}^{\circ}$ is the total molar concentration of the counterions so that $\alpha_{C} \mathbf{C}_{i}^{\circ}$ is the molar concentration of the free counterions, and $\left(\alpha_{C}\left|Z_{S}\right| e\right)$ is the apparent (effective) charge $\left|Z_{\text {ap }}\right| e$ of the polyion partially neutralized by the condensed counterions. The electroneutrality condition implies that $\left|Z_{S}\right| \mathbf{C}_{P}^{\circ}=\left|Z_{i}\right| \mathbf{C}_{i}^{\circ}$. The equivalent conductivity $\Lambda_{\text {Poly }}$ of the polyelectrolyte is therefore defined by

$$
\Lambda_{\text {Poly }}=\frac{1000 \chi}{\left|Z_{i}\right| \mathbf{C}_{i}^{\circ}}=\alpha_{C}\left(\lambda_{P}+\lambda_{i}\right) .
$$

$\lambda_{P}$ and $\lambda_{i}$ depend on the concentration of the free counterions because of the brake effects on the moving ions (or on polyions) due to their ionic atmosphere. In general one distinguishes two different ionic friction effects [2-4, 9, 12]: (a) the electrophoretic effect which is a hydrodynamic friction on the ionic atmosphere transmitted to the central ion (or polyion) via solvent molecules, (b) the ionic relaxation effect due to the perturbation of the charge distribution of the ionic atmosphere by the external electrical field $\mathbf{E}$. This polarization effect induces on the moving central ion (or polyion) a local field $\Delta \mathbf{X}^{\text {ir }}$ opposed to E. Quantitatively, these 
two effects appear in the expression of the ionic conductivity $\lambda_{i}$ of simple ions (in our case, the counterions) via the $\Delta \lambda_{i}^{\mathrm{el}}$ corrective term and the $\beta_{i}^{\text {ir }}$ relaxation term as follows:

$$
\begin{aligned}
\lambda_{i} & =\frac{\left(\lambda_{i}^{\circ}-\Delta \lambda_{i}^{\mathrm{el}}\right)}{\left(1+\beta_{i}^{\mathrm{ir}}\right)}, \\
\Delta \lambda_{i}^{\mathrm{el}} & =\left(\frac{\left|Z_{i}\right| F e}{6 \pi \eta d_{i}}\right) ; \quad d_{i}=R_{i}+\frac{1}{2 \Gamma_{i \mathrm{MSA}}}, \\
\Gamma_{i \mathrm{MSA}}^{2} \cong & \pi L_{B}\left\{\left(\frac{\left(Z_{i}\right)^{2} \alpha_{C} C_{i}}{\left[1+2 \Gamma_{i \mathrm{MSA}} R_{i}\right]^{2}}\right)\right. \\
& \left.+\left(\frac{Z_{S}^{2} \alpha_{C}^{2} C_{P}}{\left[1+2 \Gamma_{i \mathrm{MSA}}\left\langle R_{P}\right\rangle\right]^{2}}\right)\right\}, \\
\left\langle R_{P}\right\rangle & =\frac{L_{S}}{\operatorname{Ln}\left[g\left(R_{C}, L_{S}\right)\right]} .
\end{aligned}
$$

$\lambda_{i}^{\circ}$ is the ionic equivalent conductivity of the counterion at infinite dilution which expresses both the hydrodynamic friction, due to the viscosity $\eta$ of the solvent (Robinson and Stokes [2]), and the dielectric friction effect (Zwanzig [19]). $\lambda_{i}^{\circ}$ is determined experimentally by linear extrapolation at infinite dilution and according to the Debye-Onsager limiting equation [2], of the equivalent conductivity $\Lambda_{\mathrm{iX}}(\sqrt{ } I)$ (with the square root of the ionic strength $I$ ) of any corresponding simple electrolyte (e.g., if $i \equiv \mathrm{Na}^{+}$, we can choose $\Lambda_{\mathrm{iX}} \equiv$ $\Lambda_{\mathrm{NaCl}}$; extrapolation of $\Lambda_{\mathrm{NaCl}}$ in water at $25^{\circ} \mathrm{C}$ leads to $\lambda_{\mathrm{Na}^{+}}^{\circ}=$ $50.1 \mathrm{~cm}^{2} \cdot \Omega^{-1} \cdot$ eqv $_{\mathrm{Na}^{+}}{ }^{-1}$ ). The term $F$ is the Faraday, $R_{i}$ is the effective radius of the solvated counterion " $i$ ", $d_{i}$ is the radius of its ionic atmosphere, and $\Gamma_{i \mathrm{MSA}}$ is its corresponding Debye-MSA screen parameter. Notice that $\Gamma_{i \mathrm{MSA}}$ differs from the screen parameter $\Gamma_{P \mathrm{MSA}}$ relating to the polyion, because considering the high repulsion between polyions, we have assumed that the ionic atmosphere of the polyions is constituted only by free counterions; on the contrary, the ionic atmosphere of a counterion encloses both polyions and counterions. $\left\langle R_{P}\right\rangle$ is the mean radius of the polyion (analog to the radius of gyration) which is also equal to the electrostatic capacitance $C_{\mathrm{AP}}$ (in c.g.s.u.e units) of the ellipsoidal (or cylindrical) polyion $[17,20]$. Finally, the explicit expression of the ionic relaxation term $\beta_{i}^{\text {ir }}$ will be examined at the end of this section because of its interdependence with the $\beta_{P}^{\text {ir }}$ term relating to the polyions.

The expression of the equivalent conductivity $\lambda_{P}$ of the polyion is more complex because its ionic equivalent conductivity at infinite dilution $\lambda_{P}^{\circ}$ expressing both hydrodynamic friction and dielectric friction effect is experimentally inaccessible. Indeed, in contrast with simple electrolytes, ionic transport behavior of polyelectrolytes is not governed by any universal limiting law [5] allowing the determination of $\lambda_{P}^{\circ}$ by an extrapolation method at infinite dilution. For this reason we decomposed the $\lambda_{P}$ expression as follows [9]:

$$
\lambda_{P}=\frac{\lambda_{P}^{\text {Henry }}}{\left(1+\beta_{P}^{\mathrm{ir}}+\beta_{P}^{\mathrm{df}}\right)}=\frac{\left(\lambda_{P}^{\mathrm{\circ Hyd}}-\Delta \lambda_{P}^{\mathrm{el}}\right)}{\left(1+\beta_{P}^{\mathrm{ir}}+\beta_{P}^{\mathrm{df}}\right)} .
$$

The justification of the above equation is the following: the external electric field $\mathbf{E}$ acting on the polyion polarizes its ionic atmosphere as well as its surrounding solvent molecules, which gives place to an ionic relaxation field $\Delta \mathbf{X}^{\text {ir }}$ and to a dielectric relaxation field $\Delta \mathbf{X}^{\mathrm{df}}$ slowing down the movement of the polyion. The velocity $\mathbf{v}$ of the polyion can thus be written in two manners:

$$
\begin{aligned}
\mathbf{v} & =u_{P} \mathbf{E}=u_{P}^{\text {Henry }}\left(\mathbf{E}+\mathbf{\Delta} \mathbf{X}^{\mathrm{ir}}+\mathbf{\Delta} \mathbf{X}^{\mathrm{df}}\right) \\
& \equiv u_{P}^{\text {Henry }} \frac{\mathbf{E}}{\left(1+\beta_{P}^{\mathrm{ir}}+\beta_{P}^{\mathrm{df}}\right)} .
\end{aligned}
$$

$u_{P}$ is the actual electrical mobility of the polyion and $u_{P}^{\text {Henry }}$ is the Henry mobility $[9,21]$ so that

$$
\lambda_{P}=F u_{P} ; \quad \lambda_{P}^{\text {Henry }}=F u_{P}^{\text {Henry }} .
$$

$\beta_{P}^{\mathrm{ir}}$ and $\beta_{P}^{\mathrm{df}}$ express, respectively, the ionic relaxation effect and the dielectric friction effect acting on the polyion. Equation (12) implies that

$$
\begin{aligned}
& \beta_{P}^{\mathrm{ir}}=\frac{\left|\Delta \mathbf{X}^{\mathrm{ir}}\right|}{\left|\mathbf{E}+\Delta \mathbf{X}^{\mathrm{ir}}+\Delta \mathbf{X}^{\mathrm{df}}\right|}, \\
& \beta_{P}^{\mathrm{df}}=\frac{\left|\Delta \mathbf{X}^{\mathrm{df}}\right|}{\left|\mathbf{E}+\Delta \mathbf{X}^{\mathrm{ir}}+\Delta \mathbf{X}^{\mathrm{df}}\right|} .
\end{aligned}
$$

The explicit expression of the Henry equivalent conductivity $\lambda_{P}^{\text {Henry }}$ of the polyion is, [9],

$$
\lambda_{P}^{\text {Henry }}=\left(\frac{\alpha_{C} F e}{6 \pi \eta b_{S}}\right) \operatorname{Ln}\left[\frac{g\left(R_{C}, L_{S}\right)}{g\left(d_{P}, L_{S}\right)}\right] .
$$

Accordingly, we can separate $\lambda_{P}^{\text {Henry }}$ into two contributions: the purely hydrodynamic term $\lambda_{P}^{\text {oHyd }}$ due to the viscosity $\eta$ of the solvent, and the so-called electrophoretic term $\Delta \lambda_{P}^{\mathrm{el}}$, so that

$$
\begin{aligned}
& \lambda_{P}^{\circ \mathrm{Hyd}}=\left(\frac{\alpha_{C} F e}{6 \pi \eta b_{S}}\right) \operatorname{Ln}\left[g\left(R_{C}, L_{S}\right)\right], \\
& \Delta \lambda_{P}^{\mathrm{el}}=\left(\frac{\alpha_{C} F e}{6 \pi \eta b_{S}}\right) \operatorname{Ln}\left[g\left(d_{P}, L_{S}\right)\right] .
\end{aligned}
$$

It is remarkable to underline that the expression of $\lambda_{P}^{\circ \mathrm{Hyd}}$ coincides with the Hubbard-Douglass general relation [20] expressing the hydrodynamic mobility: $u_{P}^{\circ \mathrm{HD}} \equiv \lambda_{P}^{\circ \mathrm{HD}} / F$ of an arbitrarily shaped unspecified macroion of $Z_{S} e$ charge, in terms of its capacitance $C_{\mathrm{AP}}$ (generalization of Stokes' law):

$$
\begin{gathered}
u_{P}^{\circ \mathrm{HD}}=\frac{\left|Z_{S}\right| e}{6 \pi \eta C_{\mathrm{AP}}}, \\
C_{\mathrm{AP}}=\frac{L_{S}}{\operatorname{Ln}\left[g\left(R_{C}, L_{S}\right)\right]}=\left\langle R_{P}\right\rangle .
\end{gathered}
$$


We can therefore generalize the Hubbard-Douglass relation given by (17) to the Henry equivalent conductivity $\lambda_{P}^{\text {Henry }}$ as follows [17]:

$$
\lambda_{P}^{\text {Henry }}=\frac{\alpha_{C}\left|Z_{S}\right| F e}{6 \pi \eta C_{\mathrm{AP}}^{\prime}} .
$$

$C_{\mathrm{AP}}^{\prime}$ is now the electrostatic Gouy capacitance (in c.g.s.u.e units) of the ellipsoidal (or cylindrical) capacitor constituted by the polyion and by its ionic atmosphere of mean radius $\left\langle d_{P}\right\rangle$ :

$$
\left(C_{\mathrm{AP}}^{\prime}\right)^{-1}=\left[\left\langle R_{P}\right\rangle^{-1}-\left\langle d_{P}\right\rangle^{-1}\right], \quad\left\langle d_{P}\right\rangle=\frac{L_{S}}{\operatorname{Ln}\left[g\left(d_{P}, L_{S}\right)\right]} .
$$

Notice that the relative importance of the electrophoretic effect can be evaluated by the ratio:

$$
\frac{\Delta \lambda_{P}^{\mathrm{el}}}{\lambda_{P}^{\mathrm{oHyd}}}=\frac{\left\langle R_{P}\right\rangle}{\left\langle d_{P}\right\rangle}
$$

This last equation implies that the electrophoretic effect vanishes in the range of highly dilute solutions, that is, when $\left\langle d_{P}\right\rangle \rightarrow \infty$. It is also interesting to notice that certain authors [22] have described the electrophoretic mobility of polyions in polyelectrolyte solutions by means of the Debye-Onsager-MSA approach using the mean spherical approximation for the coil conformation of the polyion chain. The corresponding spherical hydrodynamic radius $R_{H}$ was evaluated according to Stokes-Einstein relation: $R_{H}=$ $k_{B} T / 6 \pi \eta D_{P}^{\circ}$, where $D_{P}^{\circ}$ is the self diffusion coefficient of the polyion at high dilution. The same spherical approximation could be used for the calculation of the ionic relaxation effect using Onsager relation applicable to spherical simple ions [3], therefore

$$
\beta_{P}^{\mathrm{ir}} \cong \frac{\alpha_{C}\left|Z_{i} Z_{S}\right| L_{B} q_{r}^{2}}{\left[3\left(1+q_{r}\right)\left\langle d_{P}\right\rangle\right]}
$$

with: $q_{r} \cong 1$, if we assume that the ionic atmosphere is free of polyions. However, Manning has demonstrated that for infinite rod-like model $\left(L_{S} \gg d_{P}\right), \beta_{P}^{\text {ir }}$ remains sensibly constant, equal to 0.13 . In order to conciliate the two results into a general expression one of the authors has proposed the following relation $[9,10]$ :

$$
\beta_{P}^{\text {ir }}=\frac{\alpha_{C}\left|Z_{S} Z_{i}\right| L_{B}\left(3 d_{P}^{2}+L_{S}^{2} / 4\right)}{\left[18\left(d_{P}^{2}+L_{S}^{2} / 4\right)^{3 / 2}\right]},
$$

so that when $L_{S} / d_{P} \rightarrow 0$, then $\beta_{P}^{\text {ir }} \rightarrow \alpha_{C}\left|Z_{i} Z_{S}\right| L_{B} /\left(6 d_{P}\right)$; this limiting expression converges toward the Debye-Onsager relation concerning spherical ions. In contrast, for polyions of large length, $L_{S} \gg d_{P}, \beta_{P}^{\text {ir }} \rightarrow 1 / 9$ for all $\mathbf{C}_{i}^{\circ}$ if $\alpha_{C} \rightarrow \alpha_{M}$.

On the other hand, according to linear irreversible thermodynamics (T.I.P) the different $\beta_{k}^{\text {ir }}$ relaxation terms of all the " $k$ " species (ions or polyions) in solution are interdependent via the general relation $[9,14]: \sum_{k} Z_{k} C_{k} \beta_{k}^{\text {ir }}=$ 0 , with $\sum_{k} Z_{k} C_{k}=0$. This means that in the case of our binary system the two relaxation terms $\beta_{P}^{\text {ir }}$ and $\beta_{i}^{\text {ir }}$, respectively, of the polyions and the counterions are equal:

$$
\beta_{P}^{\text {ir }}=\beta_{i}^{\text {ir }}
$$

Lastly, because of the importance of the dielectric friction effect on a stretched polyion (which is the main subject of this paper) the friction term $\beta_{P}^{\mathrm{df}}$ will be discussed in detail in the next paragraph.

\subsection{Importance of the Dielectric Friction Effect on a Stretched} Polyion. The aim of the present paragraph is to evaluate succinctly the frictional force on a slowly moving polyion due to dielectric loss in its surrounding medium. In fact, this dielectric friction effect depends on the conformation (shape) of the polyion. In order to show the link with previous works, we will start by presenting the general formal treatment adopted in all cases; hence we will recall the computation results relating to the spherical and ellipsoidal models. Then, we will treat, without going into the mathematical details, the specific case of a stretched polyion modeled as a chain of $\left|Z_{S}\right|$ identical charged spheres, each one having a charge $q_{n}=\alpha_{C} e$ and a radius $R_{g}$ (a linear discontinuous distribution of ionized groups).

The general mechanism of dielectric friction is the following: when a sphere of charge $q$ and radius $R_{S}$ is submitted to a moderate external alternating field $\mathbf{E}$ along the $z$ axis, it acquires a velocity $\mathbf{v}=u \mathbf{E}$, where $u$ is its electrical mobility. We indicate by $\mathbf{r}_{q}(t)=\mathbf{v} t$ the position of the center of the sphere at time $t$. During its movement the charge $q$ induces at each point $M$ of the dielectric medium (solvent) defined by its radius-vector $\mathbf{r}(x, y, z, t)$ a time-dependent polarization $\mathbf{P}(\mathbf{r}, t)$ which is proportional to the displacement field $\mathbf{D}(\mathbf{r}, t-$ $t_{1}$ ) created by $q$ at different anterior times $\left(t-t_{1}\right)$ :

$$
\mathbf{D}\left(\mathbf{r}, t-t_{1}\right)=\frac{q \mathbf{r}^{\prime}}{\left(r^{\prime}\right)^{3}}, \quad \mathbf{r}^{\prime}=\left[\mathbf{r}(x, y, z, t)-\mathbf{r}_{q}\left(t-t_{1}\right)\right] .
$$

The module $r^{\prime}$ is the distance between the point $M$ and the center of the sphere at time $\left(t-t_{1}\right)$. This noninstantaneous response results from the fact that each solvent molecule needs a relaxation time $\tau$ to be oriented along the radial field D. Mathematically, the linear relation between $\mathbf{P}(\mathbf{r}, t)$ and $\mathbf{D}\left(\mathbf{r}, t-t_{1}\right)$ is given by the following convolution integral $[17,19]$ :

$$
\mathbf{P}(\mathbf{r}, t)=\left(\frac{1}{4 \pi}\right) \int_{0}^{\infty} \gamma\left(t_{1}\right) \mathbf{D}\left(\mathbf{r}, t-t_{1}\right) d t_{1}
$$

with

$$
\begin{aligned}
\gamma(t)= & {\left[1-\left(\frac{1}{\varepsilon_{\infty}}\right)\right] \delta(t) } \\
& +\left[\left(\varepsilon_{0}-\varepsilon_{\infty}\right)\left(\tau \varepsilon_{\infty}^{2}\right)^{-1} \exp \left(\frac{-\varepsilon_{0} t}{\varepsilon_{\infty} \tau}\right)\right] .
\end{aligned}
$$


$\gamma(t)$ is the after effect function which depends on the delta function $\delta(t)$ representing electronic relaxation and on the permittivities $\varepsilon_{0}$ and $\varepsilon_{\mathrm{O}}$, respectively, the static and the highfrequency dielectric constants of the solvent. For water at $25^{\circ} \mathrm{C}, \varepsilon_{0}=78.3$ and $\varepsilon_{\infty}=1.77$. Note that we have set the upper limit of the above integral to $\infty$, because in general the dielectric relaxation time $\tau$ is small by comparison to t so that $\gamma(t) \rightarrow 0$ (vanishes rapidly) when $t>\tau$.

In turn, this induced polarization $\mathbf{P}(\mathbf{r}, t)$ exerts back on the charged sphere a resulting dielectric frictional force $\mathbf{F}^{\mathrm{df}}=$ $q \Delta \mathbf{X}^{\mathrm{df}}$ where $\boldsymbol{\Delta} \mathbf{X}^{\mathrm{df}}$ is the so called dielectric relaxation field having a direction opposed to the external field $\mathbf{E}$. The general integral relation between the $z$ component $\Delta X_{z}^{\mathrm{df}}$ of $\Delta \mathbf{X}^{\mathrm{df}}$ and the $D_{x}, D_{y}, D_{z}$ components of $\mathbf{D}\left(\mathbf{r}, t-t_{1}\right)$ via $\mathbf{P}(\mathbf{r}, t)$ and therefore $\gamma\left(t_{1}\right)$ is, [17],

$$
\Delta X_{z}^{\mathrm{df}}=\left(\frac{1}{4 \pi}\right) \int_{0}^{\infty} \gamma\left(t_{1}\right) I_{z}\left(t_{1}\right) d t_{1} .
$$

$I_{z}\left(t_{1}\right)$ is the so called key integral defined by

$$
\begin{aligned}
I_{z}\left(t_{1}\right)=\iiint\left[\frac{-D_{z}\left(t-t_{1}\right)}{r^{\prime 3}}+\frac{3 Z^{2} D_{z}\left(t-t_{1}\right)}{r^{5}}\right. \\
+\frac{3 x Z D_{x}\left(t-t_{1}\right)}{r^{\prime^{5}}} \\
\left.+\frac{3 y Z D_{y}\left(t-t_{1}\right)}{r^{\prime 5}}\right] d x d y d z,
\end{aligned}
$$

where $Z=z-v t$. Integration is taken over the whole volume except the finite region including the charged sphere (or the polyion in general) from which the dielectric medium is excluded.

It is obvious that no dielectric friction occurs, $\Delta X_{z}^{\mathrm{df}} \rightarrow 0$ when $\mathbf{v} \rightarrow 0$ (immobile sphere) or when $\tau \rightarrow 0$ (instantaneous response so that $\gamma(t) \rightarrow 0$ ). In other words, the delay effect $(\tau \neq 0)$ causes a perturbation of the equilibrium distribution of solvent molecules around the moving sphere and therefore leads to a nonsymmetrical polarization responsible of the resulting dielectric relaxation field: $\Delta X_{z}^{\mathrm{df}} \neq 0$. Consequently, linearity between causes and response implies that $\Delta \mathbf{X}^{\mathrm{df}} \sim(\mathbf{v} \tau)$. On the other hand, as the dimension of the electrical relaxation force $\mathbf{F}^{\mathrm{df}}=q \Delta \mathbf{X}^{\mathrm{df}}$ is $\sim(q)^{2} /[\text { length }]^{2}$, scaling analysis yields to $\mathbf{F}^{\mathrm{df}} \sim-(q)^{2}(\mathbf{v} \tau) / R_{S}^{3}$.

More rigorous derivations of the expression of the dielectric frictional force on a charged sphere were performed successively by Zwanzig [19], Hubbard and Onsager [23], and Wolynes [24]. In particular, if the charged sphere of large radius $R_{S}$ is assumed to be a conductor then, hydrodynamic effects become small and all theories reduce to Zwanzig's original result [19] which can be derived from (27) and (28) following the substituting of the explicit expression of $\mathbf{D}(\mathbf{r}, t-$ $t_{1}$ ) given by (25) into (29):

$$
\Delta \mathbf{X}^{\mathrm{df}}=\frac{ \pm(2 q / 3)\left[1-\varepsilon_{\mathrm{o}} / \varepsilon_{o}\right](\mathbf{v} \tau)}{\left(\varepsilon_{o} R_{S}^{3}\right)} .
$$

We can use the above equation to compute the dielectric friction effect on a spherical polyion of effective charge $q=$ $e \alpha_{S} Z_{S}$ and radius $R_{S}$. Indeed, according to (12) and (14), its velocity $\mathbf{v}$ is given by $\mathbf{v}=u_{P}^{\text {Henry }}\left(\mathbf{E}+\Delta \mathbf{X}^{\text {ir }}+\Delta \mathbf{X}^{\mathrm{df}}\right)$ with $u_{P}^{\text {Henry }}=\lambda_{P}^{\text {Henry }} / F$ and the relative dielectric friction effect is defined by $\beta_{P}^{\mathrm{df}}=\left|\boldsymbol{\Delta} \mathbf{X}^{\mathrm{df}}\right| /\left|\mathbf{E}+\Delta \mathbf{X}^{\mathrm{ir}}+\boldsymbol{\Delta} \mathbf{X}^{\mathrm{df}}\right|$, therefore (30) leads to $\beta_{P}^{\mathrm{df}}=\left(2 e \alpha_{S}\left|Z_{S}\right| / 3 F\right)\left[1-\varepsilon_{\mathrm{o}} / \varepsilon_{o}\right]\left(\lambda_{P}^{\text {Henry }} \tau\right) /\left(\varepsilon_{o} R_{S}^{3}\right)$. If we explicit the expression of $\lambda_{P}^{\text {Henry }}$ according to (19) and after replacing the relaxation time $\tau$ by its Debye expression in terms of the solvent radius $R_{w}\left(R_{\mathrm{H}_{2} \mathrm{O}}=1.4\right)$ and the viscosity $\eta$ so that $\tau=6 \pi \eta R_{w}^{3} / k_{B} T$, we obtain

$$
\beta_{P}^{\mathrm{df}}=\left(\frac{2}{3}\right)\left(\alpha_{C}\left|Z_{S}\right|\right)^{2}\left(\frac{R_{w}}{R_{S}}\right)^{3}\left(\frac{L_{B}}{C_{\mathrm{AP}}^{\prime}}\right)\left[1-\frac{\varepsilon_{\mathrm{O}}}{\varepsilon_{o}}\right] .
$$

More recently, authors of [17] have demonstrated that this last expression remains valid even in the case of an ellipsoidal polyion of minor axis $R$, interfocuses distance $L$, and effective charge $q=e \alpha_{E} Z_{S}$ but with the proviso of replacing the spherical radius $R_{S}$ by an apparent ray $R_{\text {app }}$ which is a function of the eccentricity $\gamma=L / 2 R$ so that $R_{\text {app }} \approx\langle R\rangle$ for $\gamma<1$ and $R_{\text {app }} \approx L / 2$ for $\gamma>1$.

Now, in the case of stretched chain of $\left|Z_{S}\right|$ successive charged spheres of charge $q_{n}=-e \alpha_{C}$ and of radius $R_{n}=R_{g}$ ( $n=1,\left|Z_{S}\right|$ ), each sphere $n$ moving along direction $z$ with velocity $\mathbf{v}_{p}$ undergoes from the polarized solvent molecules a dielectric frictional force $\mathbf{F}_{\mathbf{n}}^{\mathrm{df}}=q_{\mathbf{n}} \boldsymbol{\Delta} \mathbf{X}_{\mathbf{n}}^{\mathrm{df}}$, where $\boldsymbol{\Delta} \mathbf{X}_{\mathbf{n}}^{\mathrm{df}}$ is the local dielectric relaxation field. Because of the axial symmetry of the system around the $z$ axis, only the $z$ component $\Delta X_{n, z}^{\mathrm{df}}$ of $\boldsymbol{\Delta} \mathbf{X}_{\mathbf{n}}^{\mathrm{df}}$ is different from zero:

$$
\Delta X_{n, z}^{\mathrm{df}}=\left(\frac{1}{4 \pi}\right) \int_{0}^{\infty} \gamma\left(t_{1}\right) I_{n, z}\left(t_{1}\right) d t_{1}
$$

The corresponding key integral $I_{n, z}\left(t_{1}\right)$ is defined by

$$
\begin{aligned}
I_{n, z}\left(t_{1}\right)=\iiint\left[\frac{-D_{z}\left(t-t_{1}\right)}{r_{n}^{\prime 3}}+\frac{3 Z_{n}^{2} D_{z}\left(t-t_{1}\right)}{r_{n}^{\prime 5}}\right. \\
+\frac{3 x Z_{n} D_{x}\left(t-t_{1}\right)}{r_{n}^{\prime 5}} \\
\left.+\frac{3 y Z_{n} D_{y}\left(t-t_{1}\right)}{r_{n}^{\prime 5}}\right] \times d x d y d z .
\end{aligned}
$$

$x, y$, and $Z_{n}$ are the components of the vector $\mathbf{r}_{\mathbf{n}}^{\prime}$ separating a point $M(x, y, z)$ of the dielectric medium and the position of the charge $q_{\mathrm{n}}$ at time $\left(t-t_{1}\right)$. The principal difference between (25)-(29) relating to a spherical polyion and (32) and (33), comes primarily from the fact that the expression 
of the displacement field $\mathbf{D}\left(\mathbf{r}, t-t_{1}\right)$ created by the stretched chain of charged spheres at $M(x, y, z)$ is now

$$
\begin{gathered}
\mathbf{D}\left(\mathbf{r}, t-t_{1}\right)=\frac{q_{\mathbf{n}} r_{n}^{\prime}}{\left(r_{n}^{\prime}\right)^{3}}+\sum_{i \neq n}^{\left|Z_{S}\right|-1} \mathbf{D}_{i}\left(\mathbf{r}, t-t_{1}\right), \\
\mathbf{D}_{i}\left(\mathbf{r}, t-t_{1}\right)=\frac{q_{\mathbf{i}} r_{i}^{\prime}}{\left(r_{i}^{\prime}\right)^{3}} .
\end{gathered}
$$

This equation results from the principle of superposition so that (34) differs from (25) by the sum $\sum \mathbf{D}_{i}\left(\mathbf{r}, t-t_{1}\right)$ expressing the interference of the displacement field $\mathbf{D}_{n}\left(\mathbf{r}, t-t_{1}\right)$ created at $M$ by $q_{\mathrm{n}}$ at an anterior time $\left(t-t_{1}\right)$, with the different $\mathbf{D}_{i}\left(\mathbf{r}, t-t_{1}\right)$ due to the $\left(\left|Z_{S}\right|-1\right)$ charges $q_{\mathbf{i}}$ of the moving chain. Notice however that, if the distance: $b_{S}=L_{S} /\left|Z_{S}\right|$, between two successive charged groups, is sufficiently large $\left(b_{S} \gg 2 R_{g}\right.$, i.e., $f=b_{S} / R_{g} \gg 2$ ) so that: $r_{i}^{\prime}>r_{n}^{\prime}$, then the dielectric friction undergone by the sphere $q_{\mathbf{n}}$ is essentially due to polarized solvent molecules of its entourage. We could therefore neglect the interference effect and $\mathbf{D}\left(\mathbf{r}, t-t_{1}\right)$ is reduced thus to $\mathbf{D}_{n}\left(\mathbf{r}, t-t_{1}\right)=q_{\mathbf{n}} \mathbf{r}_{\mathbf{n}}^{\prime} /\left(r_{n}^{\prime}\right)^{3}$; in other words, each charged sphere of the moving polyion of radius $R_{g}$ and charge $q_{n}=-e \alpha_{C}$ behaves as if it were alone to polarize the dielectric medium. Consequently, simple direct application of Zwanzig's original result leads by analogy to (31) to the following obvious equation:

$$
\beta_{P}^{\mathrm{df}}=\left(\frac{2}{3}\right) \alpha_{C}^{2}\left(\frac{R_{w}}{R_{g}}\right)^{3}\left(\frac{L_{B}}{b_{S}}\right)\left(\frac{L_{S}}{C_{\mathrm{AP}}^{\prime}}\right)\left[1-\frac{\varepsilon_{\mathrm{O}}}{\varepsilon_{o}}\right] .
$$

Recent calculations [18] based on (14), (27), and (32)-(34) including the interference effect lead to the following corrected expression $\beta_{P}^{\mathrm{df}}$ in replacement to the above expression of $\beta_{P}^{\mathrm{df}}$ :

$$
\begin{aligned}
\beta_{P}^{\mathrm{dfi}}= & \left(\frac{2}{3 f^{3}}\right) \alpha_{C}^{2}\left(\frac{R_{w}}{R_{g}}\right)^{3}\left(\frac{L_{B}}{b_{S}}\right)\left(\frac{L_{S}}{C_{\mathrm{AP}}^{\prime}}\right) \\
& \times\left[1-\frac{\varepsilon_{\infty}}{\varepsilon_{o}}\right]\left|\left(f^{3}-4.808\right)+\frac{6.58}{\left|Z_{S}\right|}\right|, \\
f= & \frac{b_{S}}{R_{g}} .
\end{aligned}
$$

Notice that (35) and (36) are valid only in the case of slowly moving polyions so that $v_{P} \tau \leq R_{g}$. The evaluation of the interference effect in terms of the "interference parameter" $f=b_{S} / R_{g} \geq 2$ is quantified by the ratio between $\beta_{P}^{\mathrm{dfi}}$ in presence of interference and $\beta_{P}^{\mathrm{df}}$ in absence of interference:

$$
\frac{\beta_{P}^{\mathrm{dfi}}}{\beta_{P}^{\mathrm{df}}}=\left|\left(1-\frac{4.808}{f^{3}}\right)+\frac{6.58}{f^{3}\left|Z_{S}\right|}\right| \leq 1, \quad f \geq 2 .
$$

This equation shows that for a discontinuous charge distribution, that is, $b_{S} \gg R_{g}(f \gg 2)$, the interference effect becomes negligible. In contrast, it is maximal for a chain of tangent spheres $(f=2)$ and increases with $\left|Z_{S}\right|$.

It is interesting to note that $\beta_{P}^{\mathrm{dfi}}$ increases with dilution as $\alpha_{C}^{2}$ and it reaches its maximal value $\beta_{P}^{\text {odfi }}$ at infinite dilution, that is, when $\alpha_{C}=1$ (Ostwald) and $C_{\mathrm{AP}}^{\prime}=\left\langle R_{P}\right\rangle$ :

$$
\begin{aligned}
\beta_{P}^{\text {odfi }}= & \left(\frac{2}{3}\right)\left(\frac{R_{w}}{b_{S}}\right)^{3}\left(\frac{L_{B}}{b_{S}}\right)\left(\frac{L_{S}}{\left\langle R_{P}\right\rangle}\right)\left[1-\frac{\varepsilon_{\infty}}{\varepsilon_{o}}\right] \\
& \times\left|\left(f^{3}-4.808\right)+\frac{6.58}{\left|Z_{S}\right|}\right| .
\end{aligned}
$$

Finally it is important to underline the singular case of Manning's polyions $\left(R_{g} \cong 0\right.$ and $\left.L_{S} \rightarrow \infty,\left|Z_{S}\right| \rightarrow \infty\right)$. Because of the infinite length of its moving chain, the structural state of the polyion (distribution of charges, distribution of solvent molecules, and therefore $\mathbf{P}(\mathbf{r}, t))$ varies periodically with time with a period equal to: $\theta=b_{S} / v_{P}$. As for slowly moving polyions, $v_{P} \tau \approx b_{S}$, therefore $\theta \approx$ $\tau$, that is, the solvent molecules have not sufficient time to reorient themselves toward the new field $\mathbf{D}(\mathbf{r}, t)$ during the periodic variation. Consequently, the polyion seems to be immobile $\left(v_{P}=0\right)$ and thus surrounded by its initial symmetrical cylindrical distribution of solvent molecules. This conservation of the equilibrium symmetry implies the absence of any resulting dielectric relaxation field $\left(\Delta \mathbf{X}^{\mathrm{df}}=0\right)$. It is the reason for which the dielectric relaxation effect is completely absent in the restrictive case of the Manning's model.

\section{Results and Discussion}

In order to emphasize the importance of the dielectric friction on stretched polyions at high dilution we studied the conductivity behaviors of the following polyelectrolytes: sodium heparinate of high molecular weight (RB21055), sodium chondroitin sulfate, and sodium polystyrene sulphonate (NaPSS). Details of the experimental protocols of conductivity measurements are given in previous papers [9-11]. Notice that conductivity results concerning (NaPSS) are those published by Vink [14]. Comparisons for each polyelectrolyte, between experimental equivalent conductivities $\Lambda_{\text {Poly }}^{\exp }=$ $1000 \chi / \mathbf{C}_{\mathrm{Na}^{+}}^{\circ}$ and theoretical equivalent conductivities $\Lambda_{\mathrm{Poly}}^{\text {th }}=$ $\alpha_{C}\left(\lambda_{P}+\lambda_{i}\right)$ calculated in absence or in presence of dielectric friction and also in absence or in presence of interference effect, are given in Tables 1-3 and Figures 2, 3, and 4. In each table we show the different molar total concentrations $\mathbf{C}_{\mathrm{Na}^{+}}^{\circ}$ of $\mathrm{Na}^{+}$counterions, the experimental equivalent conductivities $\Lambda_{\text {Poly }}^{\text {exp }}$ in $\mathrm{cm}^{2} \cdot \Omega^{-1} \cdot$ eqv $_{\mathrm{Na}^{+}}{ }^{-1}$, the degrees of $\mathrm{Na}^{+}$condensation $\left(1-\alpha_{C}\right)$ on polyions, the apparent charge numbers $Z_{\text {ap }}=$ $\alpha_{C} Z_{S}$ of the polyions, the theoretical equivalent conductivities $\Lambda_{\mathrm{Hy}, \mathrm{El}, \mathrm{R}}$ in absence of dielectric friction (i.e., only: hydrodynamic, electrophoretic, and ionic relaxation effects), the group radius $R_{g}$, the theoretical equivalent conductivities $\Lambda_{\text {Poly }}$ in absence of interference, the theoretical equivalent conductivities $\Lambda_{\text {Poly }}^{i}$ in presence of interference, the $\%$ of the dielectric friction effect $\beta^{\text {dfi }}$ (36) in presence of interference, 
and the ratios $\beta_{P}^{\mathrm{df}} / \beta_{P}^{\mathrm{df}}$ (37) expressing the relative importance of the interference effect ( $\beta^{\mathrm{df}}$ is the dielectric friction effect in absence of interference).

3.1. Conductivity of Sodium Heparinate (RB21055). The biological polyelectrolyte sodium heparinate (RB21055) is a linear polysaccharide, well known for its anticoagulant activity. Its monomer unity is a hexasaccharide, in which each disaccharide consists in a glucosamine followed by an uronic acid. The charged groups are $\mathrm{SO}_{3}{ }^{-}\left(\mathrm{NSO}_{3}{ }^{-}\right.$or $\mathrm{OSO}_{3}{ }^{-}$) and $\mathrm{COO}^{-}$with a ratio $\mathrm{OSO}_{3}{ }^{-} / \mathrm{COO}^{-} \approx 7 / 3$. This heparin is provided by Sigma as a sodium salt extracted from pork stomach. The physical characteristics of the Sodium heparinate (RB21055) are as follows:

$M_{W} \approx 10000 \mathrm{~g} \cdot \mathrm{mol}^{-1}$ is the average molecular weight of the Sodium Heparinate (RB21055).

$Z_{S}=-53 \pm 2$ at $\mathrm{pH} \approx 7$ is the structural charge number.

$L_{S}=160 \pm 8 \AA$ is the structural length.

$b_{S}=3.2 \pm 0.2 \AA$ is the charge-to-charge distance.

$R_{C}=10 \pm 1 \AA$ is the cylindrical radius of the polyion chain.

Table 1 shows that the variation of the degree of dissociation $\alpha_{C}$ of $\mathrm{Na}^{+}$from heparin in the concentration range: $5 \times 10^{-5} \mathrm{M}<\mathbf{C}_{\mathrm{Na}^{+}}^{\circ}<5 \times 10^{-3} \mathrm{M}$ is in conformity with the dilution principle so that $\alpha_{C}$ increases with dilution from 0.59 to 0.76 and it differs from its Manning's value $\alpha_{M}=$ $b_{S} /\left|Z_{i}\right| L_{B}=0.44$. Consequently, the apparent charge number $Z_{\text {ap }}=\alpha_{C} Z_{S}$ varies with the concentration from -37.8 for $\mathbf{C}_{\mathrm{Na}^{+}}^{\circ}=5.11 \times 10^{-5} \mathrm{M}$ to -29.5 for $\mathbf{C}_{\mathrm{Na}^{+}}^{\circ}=5.13 \times 10^{-3} \mathrm{M}$. Notice that this last value seems to be different from the value $Z_{\text {ap }}^{\prime}=-18$ obtained from electrophoretic mobility using Nernst-Einstein relation: $u_{P} / D_{P}=\left|Z_{\text {ap }}^{\prime}\right| e / k_{B} T$ with $D_{P} \approx 7 \times 10^{-7} \mathrm{~cm}^{2} \cdot \mathrm{s}^{-1}$ [22]. In fact $Z_{\text {ap }}^{\prime}$ differs from $Z_{\text {ap }}$ because it depends at the same time on ionic condensation $\left(\alpha_{C}\right)$ and on ionic friction effects via $u_{P}$. Table 1 and Figure 2 show that the experimental conductivity $\Lambda_{\mathrm{NaHRB}}^{\exp }$ of sodium heparinate (RB21055) decreases sharply from 82.6 to $61.9 \mathrm{~cm}^{2} \cdot \Omega^{-1} \cdot \mathrm{eqv}_{\mathrm{Na}^{+}}{ }^{-1}$ in the low concentration range: $5 \times 10^{-5} \mathrm{M}<\mathbf{C}_{\mathrm{Na}^{+}}^{\circ}<5 \times 10^{-4} \mathrm{M}$. Theoretically, the hydrodynamic contribution (at infinite dilution) $\Lambda_{\mathrm{NaHRB}}^{\circ \mathrm{HD}}$ to $\Lambda_{\mathrm{NaHRB}}^{\exp }$ is obtained in absence of ionic condensation $\left(\alpha_{C}=\right.$ $1)$, of ionic frictions $\left(\Delta \lambda_{P}^{\mathrm{el}}=0, \beta_{P}^{\mathrm{ir}}=0\right)$ and in absence of dielectric friction $\left(\beta_{P}^{\mathrm{df}}=0\right)$. According to (6), (10), (16), and (17), $\Lambda_{\mathrm{NaHRB}}^{\circ \mathrm{HD}}=\left(\lambda_{\mathrm{HRB}}^{\circ \mathrm{HD}}+\lambda_{\mathrm{Na}^{+}}^{\circ}\right)$ with $\lambda_{\mathrm{Na}^{+}}^{\circ}=$ $50.1, \lambda_{\mathrm{HRB}}^{\circ \mathrm{HD}}=\left|Z_{S}\right| F e / 6 \pi \eta\left\langle R_{P}\right\rangle$. The mean radius $\left\langle R_{P}\right\rangle$ of heparinate (RB21055) is calculated from (10). We found $\left\langle R_{P}\right\rangle=28.8 \AA, \lambda_{\mathrm{HRB}}^{\circ \mathrm{HD}}=159.84$, and $\Lambda_{\mathrm{NaHRB}}^{\circ \mathrm{HD}}=210 \mathrm{~cm}^{2}$. $\Omega^{-1} \cdot$ eqv $_{\mathrm{Na}^{+}}{ }^{-1}$. Table 1 shows that the dielectric friction $\left(\beta_{P}^{\mathrm{dfi}}>\right.$ $50 \%$ ) is the most significant retarding effect by comparison to the electrophoretic effect and the ionic relaxation effect even when taking into account the interference of the local displacement fields. Now, as $\beta_{P}^{\mathrm{dfi}}$ is proportional to $\left(\alpha_{C}\right)^{2}$ then $\beta_{P}^{\text {dii }} \approx\left(\alpha_{C}\right)^{2} \beta_{P}^{\text {odfi }}$, which means that $\beta_{P}^{\text {dfi }}$ increases with dilution toward its maximal value $\beta_{P}^{\text {odfi }}$ at infinite dilution $\left(\alpha_{C}=1\right)$. According to (38), $\beta_{P}^{\text {odfi }}$ depends on the interference factor $f=b_{S} / R_{g}$. Adjustment between experimental conductivities $\Lambda_{\mathrm{NaHRB}}^{\exp }$ and theoretical conductivities $\Lambda_{\mathrm{NaHRB}}^{i}$ of heparinate (RB21055) leads to a group radius equal to $R_{g}=$ $1.65 \pm 0.05 \AA$ so that $f \approx 2$ (a succession of tangent charged spheres). The maximal value $\beta_{P}^{\text {odfi }}$ is therefore equal to $\beta_{P}^{\text {odfi }}=$ $(2 / 3)\left(R_{w} / b_{S}\right)^{3}\left(L_{B} / b_{S}\right)\left(L_{S} /\left\langle R_{P}\right\rangle\right)\left[1-\varepsilon_{\infty} / \varepsilon_{o}\right] \mid\left(f^{3}-4.808\right)+$ $6.58 / \mid Z_{S} \|=2.25$, for $b_{S}=3.2 \AA, f \approx 2, L_{S}=160 \AA$ and $R_{w}=1.4 \AA$. Consequently, the correct limiting conductivity at infinite dilution $\Lambda_{\mathrm{NaHRB}}^{\circ}$ must take into account the limiting dielectric friction effect $\beta_{P}^{\text {odfi }}$ in addition to the hydrodynamic friction as follows: $\Lambda_{\mathrm{NaHRB}}^{\circ}=\lambda_{\mathrm{Na}^{+}}^{\circ}+\lambda_{\mathrm{HRB}}^{\mathrm{HD}} /\left(1+\beta_{P}^{\text {odf }}\right)=$ $50.1+49.2=99.3 \mathrm{~cm}^{2} \cdot \Omega^{-1} \cdot$ eqv $_{\mathrm{Na}^{+}}{ }^{-1}$. This $\Lambda_{\mathrm{NaHRB}}^{\circ}$ is experimentally inaccessible because of the nonexistence of a universal limiting law allowing a rigorous extrapolation of $\Lambda_{\mathrm{HRB}}^{\exp }$ at infinite dilution. This impossibility is due primarily to ionic condensation effect. However, according to (6), (7), and (11), and after neglecting ionic friction effects, we can derive the following approximate complex relation between $\Lambda_{\mathrm{NaHRB}}^{\exp }$ and $\alpha_{C}$ applicable in the range of very dilute solutions for which the stretched conformation is favored and for which the electrophoretic effects and ionic relaxation effects are negligible:

$$
\begin{aligned}
\Lambda_{\mathrm{NaHRB}}^{\exp } & \approx \alpha_{C}\left[\lambda_{\mathrm{Na}^{+}}^{\circ}+\frac{\alpha_{C} \lambda_{\mathrm{HRB}}^{\circ \mathrm{HD}}}{\left(1+\alpha_{C}^{2} \beta_{P}^{\circ \mathrm{dfi}}\right)}\right] \\
& \approx \alpha_{C}\left[50.1+\frac{159.84 \alpha_{C}}{\left(1+2.25 \alpha_{C}^{2}\right)}\right] .
\end{aligned}
$$

This expression can be used as an indirect method to evaluate experimentally the degree of ionic condensation $\left(1-\alpha_{C}\right)$ from experimental measurements of the equivalent conductivity of the polyelectrolyte at high dilution. Calculation shows that for $\mathbf{C}_{\mathrm{Na}^{+}}^{\circ}<10^{-4} \mathrm{M}, \alpha_{\mathrm{C}} \geq 0.8$ in conformity with theoretical values ((1)-(4)) but incompatible with the Manning's value $\alpha_{M}=b_{S} / L_{B} \approx 0.44$. It is important to notice at this stage that the constancy of the condensation parameter at high dilution with $\alpha_{C}=\alpha_{M}$ would imply that $\Lambda_{\mathrm{NaHRB}}^{\exp }$ increases first with dilution then attains a "palier" (platform) in the range of very low concentrations in which $\alpha_{C}$ obeys to the Manning's model. This kind of behavior is not in general experimentally observed [14].

In order to reinforce the hypothesis of the stretched conformation at high dilution we will compare the equivalent conductivity of sodium heparinate $\Lambda_{\mathrm{NaHRB}}^{\mathrm{Sph}}$ calculated in the case of coiled conformation (quasi-spherical) having a mean radius $R_{S} \approx\left\langle R_{P}\right\rangle \approx k_{B} T / 6 \pi \eta D_{P} \approx 30 \AA$, to its corresponding experimental equivalent conductivity $\Lambda_{\mathrm{NaHRB}}^{\exp }$. According to Zwanzig-Frank notation $[19,25]$ the expression of the equivalent conductivity $\lambda_{\mathrm{HRB}}^{\mathrm{Sph}}$ of the spherical polyion at very high dilution is $\lambda_{\mathrm{HRB}}^{\mathrm{Sph}} \approx A \alpha_{\mathrm{Sph}}\left|Z_{S}\right| /\left[R_{S}+\left(\alpha_{\mathrm{Sph}}^{2} Z_{S}^{2} C / R_{S}^{3}\right)\right]$, with $A=92, C=10$ at $25^{\circ} \mathrm{C}$ in water, $R_{S}=30 \AA$, and $\left|Z_{S}\right|=53$. The equivalent conductivity of the (spherical) 


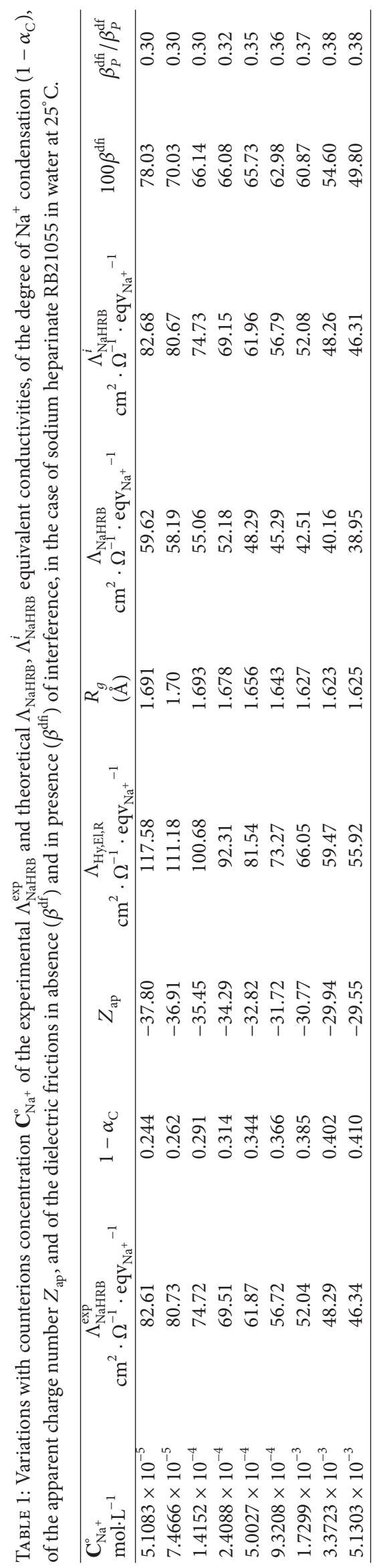




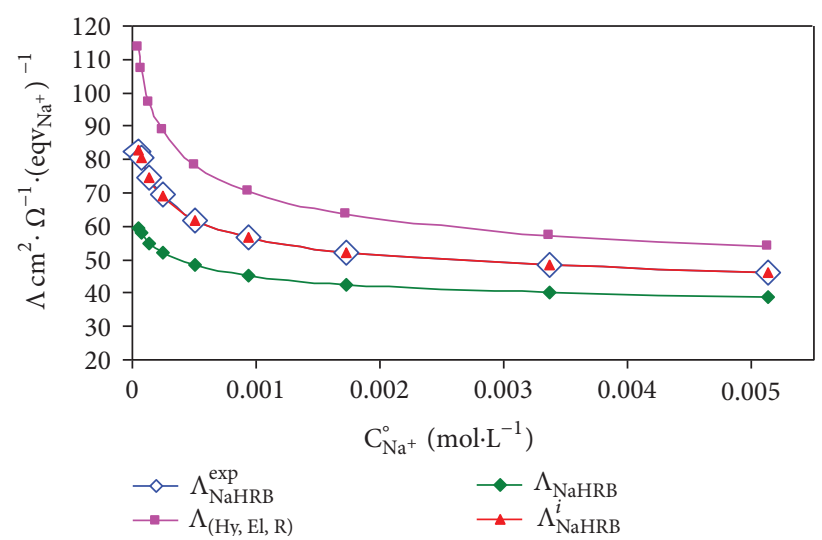

FIGURE 2: Comparison between variations with $\mathbf{C}_{\mathrm{Na}^{+}}^{\circ}$ of experimental $\left(\Lambda_{\mathrm{NaHRB}}^{\exp }\right.$, blue $\diamond$ points) and theoretical equivalent conductivities $\left(\Lambda_{\mathrm{Hy}, \mathrm{El}, \mathrm{R}}\right.$ without dielectric friction, $\Lambda_{\mathrm{NaHRB}}$ with dielectric friction in absence of interference, $\Lambda_{\mathrm{NaHRB}}^{i}$ with dielectric friction in presence of interference) of sodium heparinate RB21055 in water at $25^{\circ} \mathrm{C}$.

sodium heparinate is therefore $\Lambda_{\mathrm{NaHRB}}^{\mathrm{Sph}} \approx \alpha_{\mathrm{Sph}}\left(\lambda_{\mathrm{Na}^{+}}^{\circ}+\lambda_{\mathrm{HRB}}^{\mathrm{Sph}}\right)$. Now, according to the ionic association theory of Fuoss [3] the degree of dissociation: $\alpha_{\mathrm{Sph}}>0.68$ for $\mathbf{C}_{\mathrm{Na}^{+}}^{\circ}<$ $10^{-4} \mathrm{M}$, therefore $\Lambda_{\mathrm{NaHRB}}^{\mathrm{Sph}}>100 \mathrm{~cm}^{2} \cdot \Omega^{-1} \cdot$ eqv $_{\mathrm{Na}^{+}}{ }^{-1}$. This expected result is at least twenty percent larger $(20 \%)$ than the experimental equivalent conductivity $\Lambda_{\mathrm{NaHRB}}^{\exp }\left(\sim 83 \mathrm{~cm}^{2}\right.$. $\Omega^{-1} \cdot$ eqv $_{\mathrm{Na}^{+}}{ }^{-1}$ ), and it therefore invalidates the hypothesis of spherical conformation for heparin RB21055 polyions at high dilution. Finally it is important to underline the following principal conclusions.

(i) The interference effect decreases by $70 \%-60 \%$ the dielectric friction on the stretched polyion. However, despite this important attenuation, the resulting dielectric friction remains the principal frictional effect $\left(\beta_{P}^{\text {dfi }}\right.$ decreases with concentration from $78 \%$ to $50 \%$ ) in comparison to ionic relaxation effect and electrophoretic effect.

(ii) The adjustment of the experimental equivalent conductivities $\Lambda_{\mathrm{NaHRB}}^{\exp }$ with the theoretical conductivities $\Lambda_{\mathrm{HRB}}^{i}$ leads to a group radius $R_{g}$ sensibly constant, of about $1.65 \pm 0.05 \AA$, that is, equal to the half of the distance $b_{S}=3.2 \pm 0.2 \AA$ between two successive spherical charged groups, so that the coherent condition: $f=b_{S} / R_{g}=$ constant $\geq 2$ is verified in the studied concentration range.

(iii) The ionic relaxation effect $\beta^{\text {ir }}$ on polyions and counterions becomes important (10\%-20\%) for $\mathbf{C}_{\mathrm{Na}^{+}}^{\circ}>$ $10^{-4} \mathrm{M}$, while $\beta^{\mathrm{ir}}$ is $<6 \%$ for highly dilute solutions. This result is not in conformity with the Manning prediction: $\beta^{\text {ir }} \rightarrow 13 \%$.

(iv) The electrophoretic effect is relatively weak for $\mathrm{Na}^{+}$ counterions $(<6 \%)$, while it decreases as expected with dilution from $28 \%$ to $4 \%$ in the case of stretched heparin polyions. (v) The degree of ionic condensation $\left(1-\alpha_{C}\right)$ of $\mathrm{Na}^{+}$on heparin RB21055 increases in \% with the concentration from $24 \%$ to $41 \%$ and differs from the Manning value: $56 \%$.

(vi) The sharp increasing with dilution of the equivalent conductivity of sodium heparinate proves that both thermodynamics behavior and electrolytic conductivity behavior of this polyelectrolyte are governed by the Ostwald concentration regime despite the stretched conformation of heparin polyions.

3.2. Conductivity of Sodium Chondroitin Sulfate. The biological polyion chondroitin sulfate is a large linear polysaccharide composed of repeating disaccharide units altering an amino sugar $N$-acetyl- $\beta$-galactosamine- 4 -sulfate and an $\beta$ glucuronic acid. The sulfate groups as well as the uronic acids result in linear chains having a negative charge. Chondroitin sulfate is provided by Sigma as a sodium salt from bovine trachea. The physical characteristics of the macroion are [10, 11] as follows:

$M_{W} \approx 21430 \mathrm{~g} \cdot \mathrm{mol}^{-1}$ is the average molecular weight of the used polyelectrolyte.

$Z_{S}=-75 \pm 3$ is the structural charge number.

$L_{S}=435 \pm 15 \AA$ is the structural length.

$b_{S}=5.8 \pm 0.2 \AA$ is the charge-to-charge distance.

$R_{C}=6 \pm 0.5 \AA$ is the cylindrical radius of the polyion chain.

This polyion is therefore about three times longer than heparin $\mathrm{RB}$, and regarding its significant charge separation $b_{S}$, it presents a more discontinuous linear charge distribution. On the other hand, according to Manning's theory, we expect a weaker degree of condensation $\left(1-\alpha_{C}\right)$ despite the relative importance of its structural charge number. Table 2 shows that the degree of dissociation $\alpha_{C}$ of $\mathrm{Na}^{+}$from chondroitin increases slightly with dilution from 0.804 to 0.852 in the concentration range: $1.38 \times 10^{-4} \mathrm{M}<\mathbf{C}_{\mathrm{Na}^{+}}^{\circ}<$ $1.11 \times 10^{-3} \mathrm{M}$. Sodium chondroitin sulfate is one of peculiar polyelectrolytes for which the behavior of ionic condensation in aqueous solution is compatible at the same time with the model of Manning and with the principle of dilution $[10,11]$. Indeed, the Manning's value of the condensation parameter is $\alpha_{M}=b_{S} /\left|Z_{i}\right| L_{B}=0.81$. Consequently, the apparent charge number $Z_{\text {ap }}=\alpha_{C} Z_{S}$ varies slightly with the concentration from -63.9 for $\mathbf{C}_{\mathrm{Na}^{+}}^{\circ}=1.38 \times 10^{-4} \mathrm{M}$ to -60.3 for $\mathbf{C}_{\mathrm{Na}^{+}}^{\circ}=$ $1.11 \times 10^{-3} \mathrm{M}$.

Table 2 and Figure 3 show that the experimental conductivity of sodium chondroitin $\Lambda_{\mathrm{NaChondro}}^{\exp }$ increases with dilution in a monotonous way from 65.3 to $78.54 \mathrm{~cm}^{2}$. $\Omega^{-1} \cdot$ eqv $_{\mathrm{Na}^{+}}{ }^{-1}$ in the concentration range: $1.38 \times 10^{-4} \mathrm{M}<$ $\mathbf{C}_{\mathrm{Na}^{+}}^{\circ}<1.11 \times 10^{-3} \mathrm{M}$, (i.e., without the appearance of any palier). The hydrodynamic contribution (at infinite dilution) $\Lambda_{\mathrm{NaChondro}}^{\circ \mathrm{HD}}$ to $\Lambda_{\mathrm{NaChondro}}^{\exp }$ is obtained in absence of ionic condensation and in absence of other frictional effects from 


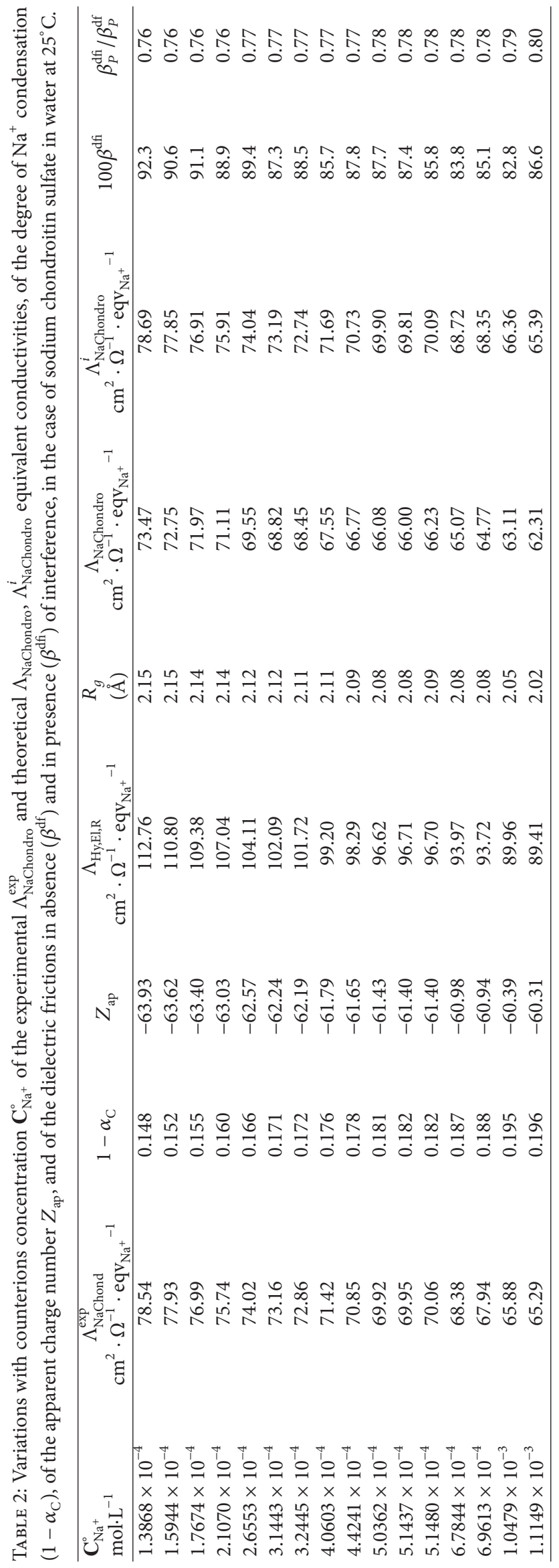




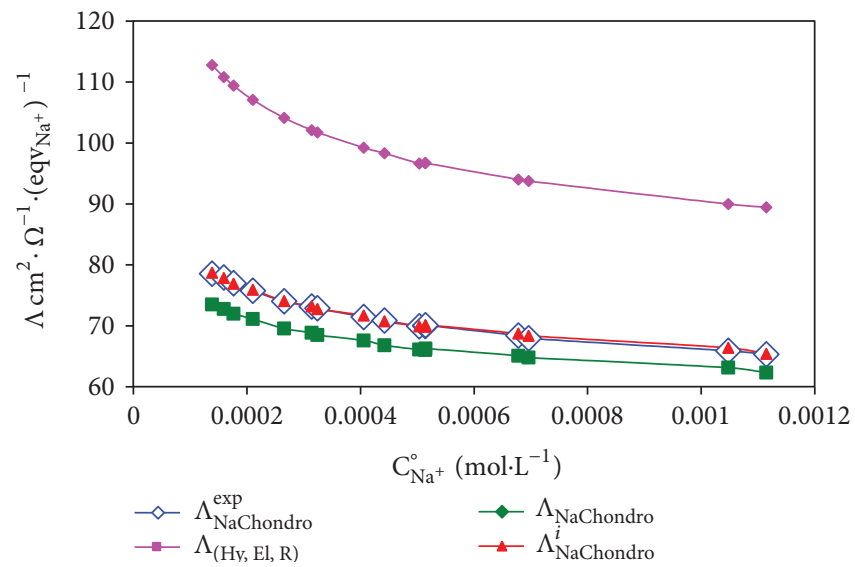

FIGURE 3: Comparison between variations with $\mathbf{C}_{\mathrm{Na}^{+}}^{\circ}$ of experimental $\left(\Lambda_{\mathrm{NaChondro}}^{\exp }\right.$, blue $\diamond$ points) and theoretical equivalent conductivities $\left(\Lambda_{\mathrm{Hy}, \mathrm{El}, \mathrm{R}}\right.$ without dielectric friction, $\Lambda_{\mathrm{NaChondro}}$ with dielectric friction in absence of interference, $\Lambda_{\mathrm{NaChondro}}^{i}$ with dielectric friction in presence of interference) of sodium chondroitin sulfate in water at $25^{\circ} \mathrm{C}$.

(6), (10), (16), and (17), so that $\Lambda_{\mathrm{NaChondro}}^{\circ \mathrm{HD}}=\left(\lambda_{\text {Chondro }}^{\circ \mathrm{HD}}+\right.$ $\lambda_{\mathrm{Na}^{+}}^{\circ}$ ) with $\lambda_{\mathrm{Na}^{+}}^{\circ}=50.1$ with $\lambda_{\text {Chondro }}^{\circ \mathrm{HD}}=\left|Z_{S}\right| \mathrm{Fe} / 6 \pi \eta\left\langle R_{P}\right\rangle$. The mean radius $\left\langle R_{P}\right\rangle$ of the chondroitin sulfate is equal to $50.8 \AA$, therefore $\lambda_{\text {Chondro }}^{\circ H D}=136.1$ and $\Lambda_{\mathrm{NaChondro}}^{\circ \mathrm{HD}}=$ $186.2 \mathrm{~cm}^{2} \cdot \Omega^{-1} \cdot \mathrm{eqv}_{\mathrm{Na}^{+}}{ }^{-1}$. Table 2 shows also that the dielectric friction $\left(\beta_{P}^{\mathrm{dfi}}>85 \%\right)$ is the most significant retarding effect by comparison to the electrophoretic effect and the ionic relaxation effect even when taking into account the interference of the local displacement fields. $\beta_{P}^{\text {dfi }}$ depends on the interference factor $f=b_{S} / R_{g}$. Adjustment between experimental and theoretical conductivities of chondroitin sulfate leads to a group radius equal to $R_{g}=2.14 \pm$ $0.2 \AA$ so that $f=b_{S} / R_{g}=2.71$ (a discontinuous linear charge distribution), the maximal value $\beta_{P}^{\text {odfi }}$ is, according to (38), equal to $\beta_{P}^{\circ}$ dfi $=(2 / 3)\left(R_{w} / b_{S}\right)^{3}\left(L_{B} / b_{S}\right)\left(L_{S} /\left\langle R_{P}\right\rangle\right)[1-$ $\left.\varepsilon_{\infty} / \varepsilon_{0}\right]\left|\left(f^{3}-4.808\right)+6.58 /\right| Z_{S} \|=1.47$ for $b_{S}=5.8 \AA$, $f \approx 2.7, L_{S}=435 \AA$ and $R_{w}=1.4 \AA$. Consequently, the new limiting $\Lambda_{\mathrm{NaChondro}}^{\circ}$ must take into account the limiting dielectric friction effect $\beta_{P}^{\circ \text { dfi }}$ in addition to the hydrodynamic friction as follows: $\Lambda_{\mathrm{NaChondro}}^{\circ}=\lambda_{\mathrm{Na}^{+}}^{\circ}+\lambda_{\mathrm{Chondro}}^{\circ \mathrm{HD}} /\left(1+\beta_{P}^{\text {odfi }}\right)=$ $50.1+36.7=86.8 \mathrm{~cm}^{2} \cdot \Omega^{-1} \cdot$ eqv $_{\mathrm{Na}^{+}}{ }^{-1}$. This $\Lambda_{\mathrm{NaChondro}}^{\circ}$ is experimentally inaccessible. However, according to our previous analysis and to (6), (7), and (11), we can proceed in the same manner that for Heparin RB21055 in order to derive the following approximate relation between the experimental equivalent conductivity of chondroitin sulfate $\Lambda_{\mathrm{NaChondro}}^{\exp }$ and the degree of dissociation $\alpha_{C}$ in the range of very dilute solutions for which the stretched conformation is favored and for which the electrophoretic effects and ionic relaxation effects are negligible, we obtain

$$
\begin{aligned}
\Lambda_{\text {NaChondro }}^{\exp } & \approx \alpha_{C}\left[\lambda_{\mathrm{Na}^{+}}^{\circ}+\frac{\alpha_{C} \lambda_{\mathrm{Chondro}}^{\circ \mathrm{HD}}}{\left(1+\alpha_{C}^{2} \beta_{P}^{\circ \text { dfi }}\right)}\right] \\
& \approx \alpha_{C}\left[50.1+\frac{136.13 \alpha_{C}}{\left(1+1.47 \alpha_{C}^{2}\right)}\right] .
\end{aligned}
$$

In conclusion we can underline the following points.

(i) The interference effect decreases by $25 \%$ the dielectric friction on stretched chondroitin sulfate polyions. However, despite this attenuation, the resulting dielectric friction remains the principal frictional effect ( $\beta_{P}^{\text {dfi }}$ decreases with concentration from $92 \%$ to $86 \%$ ) in comparison to ionic relaxation effect and electrophoretic effect.

(ii) The adjustment of experimental equivalent conductivities $\Lambda_{\text {NaChondro }}^{\exp }$ with the theoretical conductivi-

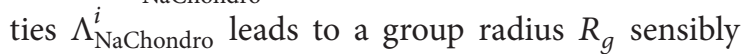
constant of about $2.13 \pm 0.2 \AA$ so that the coherent condition: $f=b_{S} / R_{g}=2.7 \geq 2$ is verified in the studied concentration range.

(iii) The ionic relaxation effect $\beta^{\text {ir }}$ on chondroitin polyions and $\mathrm{Na}^{+}$counterions remains approximately constant $(\sim 15 \%)$ in the studied range of relatively low concentrations: $1.38 \times 10^{-4} \mathrm{M}<\mathbf{C}_{\mathrm{Na}^{+}}^{\circ}<1.11 \times 10^{-3} \mathrm{M}$ in conformity with the Manning prediction.

(iv) The electrophoretic effect is relatively weak for the $\mathrm{Na}^{+}$conterions $(<4 \%)$, while it normally decreases with dilution from $27 \%$ to $12 \%$ for stretched chondroitin sulfate polyions.

(v) The degree of ionic condensation $\left(1-\alpha_{C}\right)$ of $\mathrm{Na}^{+}$ on stretched chondroitin sulfate polyions increases in $\%$ slowly with the concentration from $15 \%$ to $19.6 \%$. These values are close to the Manning value: (1 $\left.\alpha_{M}\right)=0.19$.

(vi) The weak increasing with dilution of the equivalent conductivity of sodium chondroitin sulfate proves that both thermodynamics behavior and electrolytic conductivity behavior of this polyelectrolyte at very high dilution are intermediate between the Ostwald concentration regime and the Manning concentration regime. 
3.3. Conductivity of Sodium Polystyrene Sulphonate (NaPSS). Polystyrene sulphonate is a linear chain of 4styrenesulfonic acid groups. The linear formula of NaPSS is $-\left(\mathrm{C}_{8} \mathrm{H}_{7} \mathrm{NaO}_{3} \mathrm{~S}\right)_{n}-$; it is provided by Aldrich Chemicals as a sodium salt. The physical characteristics of the studied sodium polystyrene sulphonate are as follows:

$M_{W} \approx 600000$ is the average molecular weight in $\mathrm{g} \cdot \mathrm{mol}^{-1}$

of the used polyelectrolyte. $Z_{S}=-2900 \pm 120$ is the structural charge number.

$L_{S}=7250 \pm 300 \AA$ is the structural length.

$b_{S}=2.5 \pm 0.1 \AA$ is the charge-to-charge distance.

$R_{C}=6.9 \pm 0.5 \AA$ is the cylindrical radius of the polyion chain.

Table 3 shows that the degree of dissociation $\alpha_{C}$ of $\mathrm{Na}^{+}$ from PSS decreases very slightly with dilution from 0.441 to 0.457 in the concentration range: $1.16 \times 10^{-3} \mathrm{M}<\mathbf{C}_{\mathrm{Na}^{+}}^{\circ}<$ $5.14 \times 10^{-3} \mathrm{M}$. This behavior seems in contradiction with the Ostwald regime. However this slight variation is not very significant in regard of uncertainties and we can assume that $\alpha_{C}$ remains sensibly constant. In fact, theoretical formal calculations according to (1) show that $\alpha_{C}$ presents a little minimum (0.413) for $\mathbf{C}_{\mathrm{Na}^{+}}^{\circ} \approx 10^{-5} \mathrm{M}$, then it increases slowly according to the principle of dilution until 0.605. We can therefore distinguish between two concentration ranges or regimes: (a) the Manning regime: $1 \times 10^{-5} \mathrm{M}<$ $\mathbf{C}_{\mathrm{Na}^{+}}^{\circ}<5.1 \times 10^{-3} \mathrm{M}$, in which $\alpha_{C}$ remains practically constant, and (b) a formal Ostwald concentration regime: $\mathbf{C}_{\mathrm{Na}^{+}}^{\circ} \ll 1 \times 10^{-5} \mathrm{M}$, in which $\alpha_{\mathrm{C}}$ increases with dilution. Because of the large length of PSS (7250 $\AA$ ) it is not possible to experimentally observe highly diluted regime via the increasing of conductivity. Only the Manning concentration regime was experimentally observed via the constancy of the equivalent conductivity of NaPSS during dilution in the studied concentration range. Note that the Manning regime has also been observed for NaPSS polyelectrolytes of molar masses $M_{w}$ between $8000 \mathrm{~g} \cdot \mathrm{mol}^{-1}$ and $360000 \mathrm{~g} \cdot \mathrm{mol}^{-1}$ [16]. However, despite the large length of PSS and despite the constancy of its degree of dissociation, the actual value $\sim 0.45$ of $\alpha_{C}$ is in fact different from its Manning's value $\alpha_{M}=$ $b_{S} /\left|Z_{i}\right| L_{B}=0.35$. Consequently, the apparent charge number $Z_{\text {ap }}$ varies with the concentration from -1326 for $\mathbf{C}_{\mathrm{Na}^{+}}^{\circ}=$ $5.14 \times 10^{-3} \mathrm{M}$ to -1279 for $\mathbf{C}_{\mathrm{Na}^{+}}^{\circ}=1.16 \times 10^{-3} \mathrm{M}$.

Table 3 and Figure 4 show that the experimental conductivity $\Lambda_{\mathrm{NaPSS}}^{\exp }$ decreases very slowly with concentration in a monotonous way from 35.5 to $34.9 \mathrm{~cm}^{2} \cdot \Omega^{-1} \cdot \operatorname{eqv}_{\mathrm{Na}^{+}}{ }^{-1}$ in the indicated concentration range. The mean radius $\left\langle R_{P}\right\rangle$ of the polyion is equal to $521.2 \AA$; therefore according to (16)(18) the hydrodynamic mobility $\lambda_{\text {PSS }}^{\circ \mathrm{HD}}$ of the PSS polyion is equal to $\lambda_{\text {PSS }}^{\circ \mathrm{HD}}=\left|Z_{S}\right| \mathrm{Fe} / 6 \pi \eta\left\langle R_{P}\right\rangle=512.8 \mathrm{~cm}^{2} \cdot \Omega^{-1}$. eqv $_{\mathrm{Na}^{+}}{ }^{-1}$. Adjustment between experimental and theoretical conductivities of NaPSS polyelectrolyte leads to a constant group radius equal to $R_{g} \cong 1.21 \AA$ so that $f=b_{S} / R_{g}=$ 2.06 (a quasi-continuous linear charge distribution of tangent spheres); the maximal value of $\beta_{P}^{\text {odfi }}$ is therefore $\beta_{P}^{\text {odf }}=$ $(2 / 3)\left(R_{w} / b_{S}\right)^{3}\left(L_{B} / b_{S}\right)\left(L_{S} /\left\langle R_{P}\right\rangle\right)\left[1-\varepsilon_{\infty} / \varepsilon_{0}\right] \mid\left(f^{3}-4.808\right)+$ $6.58 / \mid Z_{S} \|=17.9$ for $b_{S}=2.5 \AA, f \approx 2, L_{S}=7250 \AA$

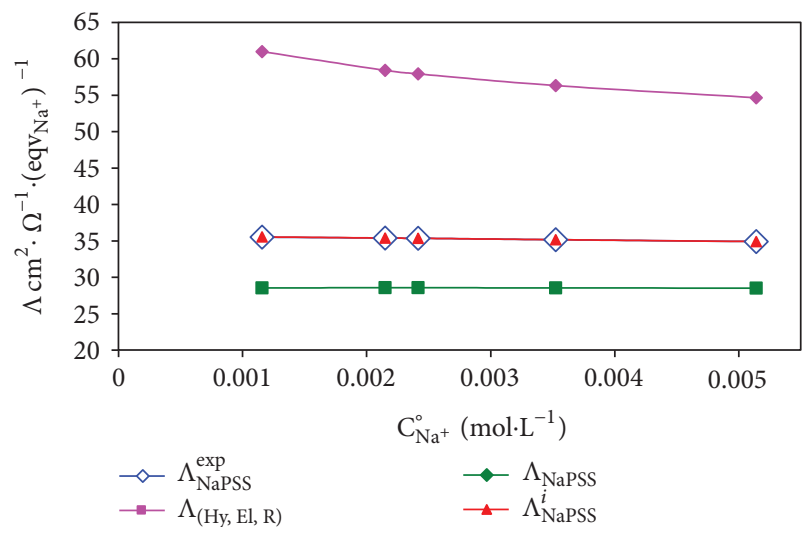

FIGURE 4: Comparison between variations with $\mathbf{C}_{\mathrm{Na}^{+}}^{\circ}$ of experimental $\left(\Lambda_{\mathrm{NaPSS}}^{\exp }\right.$, blue $\diamond$ points) and theoretical equivalent conductivities $\left(\Lambda_{\mathrm{Hy}, \mathrm{El}, \mathrm{R}}\right.$ without dielectric friction, $\Lambda_{\mathrm{NaPSS}}$ with dielectric friction in absence of interference, $\Lambda_{\text {NaPSS }}^{i}$ with dielectric friction in presence of interference) of sodium polystyrene sulphonate in water at $25^{\circ} \mathrm{C}$.

and $R_{w}=1.4 \AA$. Using our previous analysis and (6), (7), and (11), we can proceed in the same manner that for Heparin and Chondroitin in order to derive the following approximate relation between $\Lambda_{\mathrm{NaPSS}}^{\exp }$ and $\alpha_{\mathrm{C}}$, in the range of dilute solutions for which the stretched conformation is favored, we obtain

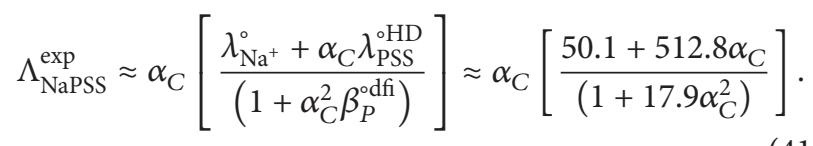

Now, as $\alpha_{C}$ undergoes weak variation in the Manning region, $\Lambda_{\mathrm{NaPSS}}^{\exp }$ remains sensibly constant (palier of $\Lambda_{\mathrm{NaPSS}}^{\exp } \sim 35 \mathrm{~cm}^{2} \cdot \Omega^{-1} \cdot \mathrm{eqv}_{\mathrm{Na}^{+}}{ }^{-1}$ ), in the concentration range: $1 \times 10^{-3} \mathrm{M}<\mathbf{C}_{\mathrm{Na}^{+}}^{\circ}<5 \times 10^{-3} \mathrm{M}$. We can however formally expect the emergence of highly diluted regime for $\mathbf{C}_{\mathrm{Na}^{+}}^{\circ}<$ $10^{-6} \mathrm{M}$. It is also important to underline that the experimental observation of Manning behavior proves the veracity of the stretched conformation. Indeed, it is well known that for spherical conformation the degree of dissociation $\alpha_{S}$ increases normally with dilution (Fuoss behavior) so that we will not be able to observe in this case any palier of conductivity.

In conclusion we can underline the following points.

(i) The interference effect decreases by $55 \%$ the dielectric friction on PSS polyions. However, despite this attenuation, and particularly in this case of very long chains, the dielectric friction remains the principal frictional effect $\left(\beta_{P}^{\text {dfi }}\right.$ decreases with concentration from $172 \%$ to $142 \%$ ) in comparison to ionic relaxation effect and electrophoretic effect.

(ii) The adjustment of experimental equivalent conductivities $\Lambda_{\mathrm{NaPSS}}^{\exp }$ with the theoretical conductivities $\Lambda_{\text {NaPSS }}^{i}$ leads to a group radius $R_{g}$ sensibly constant of about $1.21 \AA$ so that the coherent condition: 


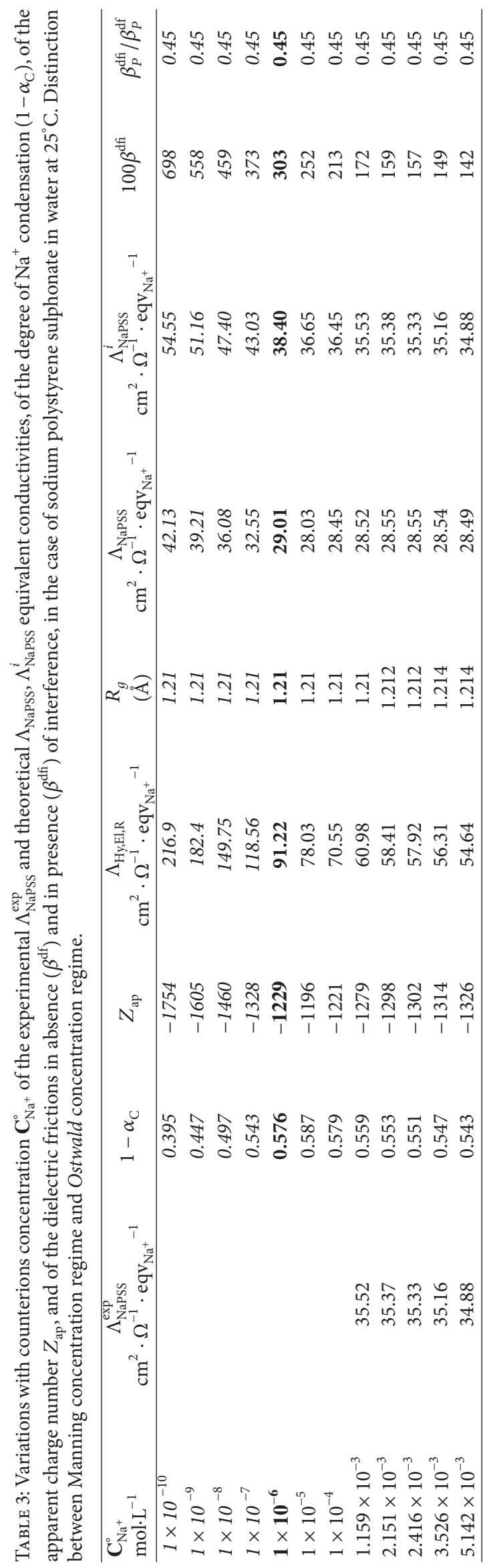


$f=b_{S} / R_{g}=2.06 \geq 2$ is verified in the studied concentration range.

(iii) The ionic relaxation effect $\beta^{\text {ir }}$ on PSS polyions and $\mathrm{Na}^{+}$counterions is relatively important and remains sensibly constant $(\sim 14 \%)$ in the studied range of relatively low concentrations: $1.16 \times 10^{-3} \mathrm{M}<\mathbf{C}_{\mathrm{Na}^{+}}^{\circ}<$ $5.14 \times 10^{-3} \mathrm{M}$. The reason of the constancy of the relaxation effect is the large chain length of PSS (7250 $\AA$ ) by comparison to the minor axis of the ionic atmosphere $d_{P}$. Indeed, $d_{P}=199 \AA$ for $\mathbf{C}_{\mathrm{Na}^{+}}^{\circ}=1.16 \times$ $10^{-3} \mathrm{M}$ and $d_{P}=98 \AA$ for $\mathbf{C}_{\mathrm{Na}^{+}}^{\circ}=5.14 \times 10^{-3} \mathrm{M}$. Therefore, $d_{P} / L_{S}<0.03$ and according to (23), $\beta_{P}^{\text {ir }} \approx$ $\left(\alpha_{C} / \alpha_{M}\right)(1 / 9) \approx 0.14$ for all $\mathbf{C}_{\mathrm{Na}^{+}}^{\circ}$.

(iv) The electrophoretic effect $\left(\Delta \lambda_{P}^{\mathrm{el}} / \lambda_{P}^{\mathrm{oHyd}}=\left\langle R_{P}\right\rangle /\left\langle d_{P}\right\rangle\right)$ on PSS polyions is more important than $\beta^{\text {ir }}$ in the studied concentration range; it decreases slowly with dilution from $61.9 \%$ to $51.7 \%$. The electrophoretic effect on PSS becomes formally negligible $(<5 \%)$ for highly dilute solution: $\mathbf{C}_{\mathrm{Na}^{+}}^{\circ}<10^{-6} \mathrm{M}$.

(v) The observed conductivity variation of NaPSS in the concentration range: $1.16 \times 10^{-3} \mathrm{M}<\mathbf{C}_{\mathrm{Na}^{+}}^{\circ}<$ $5.14 \times 10^{-3} \mathrm{M}$ reveals a behavior compatible with the Manning concentration regime because of the large length of its stretched chain.

\section{Conclusions}

In this work we have used electrolytic conductivity as a powerful tool to investigate, in dilute aqueous solutions at $25^{\circ} \mathrm{C}$, the behavior of three kinds of flexible polyelectrolytes of different chain lengths $L_{S}$ and different charge distances $b_{S}$ and without added salt: sodium heparinate $\left(L_{S}=160 \AA\right.$, $\left.b_{S}=3.2 \AA\right)$, sodium chondroitin sulfate $\left(L_{S}=435 \AA\right.$, $\left.b_{S}=5.8 \AA\right)$, and sodium polystyrene sulphonate $\left(L_{S}=\right.$ $7250 \AA, b_{S}=2.5 \AA$ ). A strong influence on their equivalent conductivities $\Lambda_{\text {Poly }}$ has been shown to exist due to the interdependent effects of $\mathrm{Na}^{+}$condensation and of dielectric friction and particularly when their chains adopt a stretched conformation at high dilute solutions. In contrast, the ionic friction effects on counterions and polyions due to the electrophoretic effects $\left(\Delta \lambda_{\mathrm{Na}^{+}}^{\mathrm{el}}, \Delta \lambda_{P}^{\mathrm{el}}\right)$ and to the ionic relaxation effect $\left(\beta^{\text {ir }}\right)$ are less important and become negligible at high dilution. The correlation between $\Lambda_{\text {Poly }}$ and, respectively, the degree of condensation $\left(1-\alpha_{C}\right)$, the limiting equivalent conductivity $\lambda_{\mathrm{Na}^{+}}^{\circ}$, the hydrodynamic equivalent conductivity of the polyion $\lambda_{P}^{\circ \mathrm{HD}}$, the limiting dielectric friction coefficient $\left(\beta_{P}^{\text {odf }}\right)$, and the ionic friction terms $\left(\Delta \lambda_{\mathrm{Na}^{+}}^{\mathrm{el}}, \Delta \lambda_{P}^{\mathrm{el}}, \beta^{\mathrm{ir}}\right)$ can be expressed by the following general expression:

$$
\begin{aligned}
\Lambda_{\text {Poly }} \approx & \alpha_{C}\left(1+\beta^{\mathrm{ir}}\right)^{-1} \\
& \times\left[\left(\lambda_{\mathrm{Na}^{+}}^{\circ}-\Delta \lambda_{\mathrm{Na}^{+}}^{\mathrm{el}}\right)+\frac{\left(\alpha_{C} \lambda_{P}^{\mathrm{oHD}}-\Delta \lambda_{P}^{\mathrm{el}}\right)}{\left(1+\alpha_{C}^{2} \beta_{P}^{\mathrm{odf}}\right)}\right] .
\end{aligned}
$$

The limiting dielectric friction coefficient $\left(\beta_{P}^{\circ \mathrm{df}}\right)$ is very sensitive to the conformation of the polyion, to the charge distance $b_{S}$, and to the radius $R_{g}$ of charged groups. Indeed, $\left(\beta_{P}^{\text {odf }}\right)$ is weak for coiled conformations and vanishes when $v_{P} \tau>R_{g}$, where $v_{P}$ is the velocity of the polyion and $\tau$ is the dielectric relaxation time of the solvent molecules. In contrast, for a stretched chain of charged spheres, $\left(\beta_{P}^{\circ \mathrm{df}}\right)$ is in general larger than $50 \%$ and increases with the interference term $f^{3}=\left(b_{S} / R_{g}\right)^{3}$ as follows:

$$
\begin{aligned}
\beta_{P}^{\text {odfi }}= & \left(\frac{2}{3}\right)\left(\frac{R_{w}}{b_{S}}\right)^{3}\left(\frac{L_{B}}{b_{S}}\right)\left(\frac{L_{S}}{\left\langle R_{P}\right\rangle}\right)\left[1-\frac{\varepsilon_{\mathrm{\infty}}}{\varepsilon_{o}}\right] \\
& \times\left|\left(f^{3}-4.808\right)+\frac{6.58}{\left|Z_{S}\right|}\right| \sim \operatorname{Ln}\left(\frac{L_{S}}{R_{C}}\right) .
\end{aligned}
$$

On the basis of our data and of our analysis, we can provisionally conclude that it is not possible to propose a universal model in order to describe the electrolytic conductivity behavior of polyelectrolytes having whichever chain lengths and distances between charged groups. However, two concentration regimes could be identified: (a) the Ostwald concentration regime (it is the case of heparinate of sodium) for which $\alpha_{C}$ and $\Lambda_{\text {Poly }}$ increase with dilution, while $\Delta \lambda_{\mathrm{Na}^{+}}^{\mathrm{el}}$, $\Delta \lambda_{P}^{\mathrm{el}}$, and $\beta^{\mathrm{ir}} \rightarrow 0$; (b) the Manning concentration regime (it is the case of sodium polystyrene sulphonate) for which $\alpha_{C}$, $\beta^{\text {ir }}$, and $\Lambda_{\text {Poly }}$ remain sensibly constant with dilution, while $\Delta \lambda_{\mathrm{Na}^{+}}^{\mathrm{el}}$ and $\Delta \lambda_{P}^{\mathrm{el}}$ decrease with concentration. It is important to underline that the ideal Manning behavior $\left(L_{S} / R_{C} \rightarrow \infty\right)$ implies the restrictive conditions: $\alpha_{C}=\alpha_{M}=b_{S} / L_{B}, \beta^{\text {ir }}=$ $1 / 9$, and $\beta_{P}^{\text {odfi }}=0$ (no dielectric friction: $v_{P} \tau>R_{g}$ ), while less restrictive conditions imply $\alpha_{C}=$ constant, $\beta^{\text {ir }}=\left(\alpha_{C} / 9 \alpha_{M}\right)$, and $\beta_{P}^{\text {odfi }} \neq 0$. Only the sodium chondroitin sulfate presents an intermediate behavior between Ostwald concentration regime and Manning concentration regime with $\alpha_{C} \approx \alpha_{M}=$ 0.81 and $\beta^{\text {ir }} \approx 0.13$. This mixed behavior is characterized by a slowly increasing of its equivalent conductivity with dilution.

\section{Acknowledgment}

The authors are grateful to the General Direction of Scientific Research of Tunisia (D.G.R.S.T) for assistance and supporting grants.

\section{References}

[1] L. Blum, "Mean spherical model for asymmetric electrolytes," Journal Molecular Physics, vol. 30, no. 5, pp. 1529-1535, 1975.

[2] R. A. Robinson and R. H. Stokes, Electrolyte Solutions, Butterworths, London, UK, 1959.

[3] R. Fuoss and F. Accascina, Electrolytic Conductance, Interscience, New York, NY, USA, 1969.

[4] P. Turq, L. Blum, O. Bernard, and W. Kunz, "Conductance in associated electrolytes using the mean spherical approximation," The Journal of Physical Chemistry, vol. 99, no. 2, pp. 822-827, 1995. 
[5] M. Muthukumar, "Dynamics of polyelectrolyte solutions," The Journal of Chemical Physics, vol. 107, no. 7, pp. 2619-2635, 1997.

[6] E. Sélégny, Polyelectrolytes, D. Reildel Publishing Company, Boston, Mass, USA, 1974.

[7] G. S. Manning, "Limiting laws and counterion condensation in polyelectrolyte solutions. 7. Electrophoretic mobility and conductance," The Journal of Physical Chemistry, vol. 85, no. 11, pp. 1506-1515, 1981.

[8] A. V. Dobrynin and M. Rubinstein, "Theory of polyelectrolytes in solutions and at surfaces," Progress in Polymer Science, vol. 30, no. 11, pp. 1049-1118, 2005.

[9] J. M'Halla, "Polyelectrolytic conductance. Limiting laws in conformity with the principles of equilibrium and nonequilibrium thermodynamics. Interdependence between conformation, condensation and dielectric friction," Journal of Molecular Liquids, vol. 82, no. 3, pp. 183-218, 1999.

[10] J. M'Halla, R. Besbes, R. Bouazzi, and S. Boughammoura, "About the singular behavior of the ionic condensation of sodium chondroitin sulfate: conductivity study in water and water-dioxane mixture," Chemical Physics, vol. 321, no. 1-2, pp. 10-24, 2006.

[11] J. M’Halla, R. Besbes, R. Bouazzi, and S. Boughammoura, "Ionic condensation of sodium chondroitin sulfate in water-dioxane mixture," Journal of Molecular Liquids, vol. 130, no. 1-3, pp. 59-69, 2007.

[12] G. S. Manning, "Limiting law for the conductance of the rod model of a salt-free polyelectrolyte solution," The Journal of Physical Chemistry, vol. 79, no. 3, pp. 262-265, 1975.

[13] N. V. Brilliantov, D. V. Kuznetsov, and R. Klein, "Chain collapse and counterion condensation in dilute polyelectrolyte solutions," Physical Review Letters, vol. 81, no. 7, pp. 1433-1436, 1998.

[14] H. Vink, "Studies of electrical transport processes in polyelectrolyte solutions," Journal of the Chemical Society, Faraday Transactions 1, vol. 85, no. 3, pp. 699-709, 1989.

[15] H. Vink, "Conductivity of polyelectrolytes in very dilute solutions," Journal of the Chemical Society, Faraday Transactions 1, vol. 77, no. 10, pp. 2439-2449, 1981.

[16] C. Wandrey, "Concentration regimes in polyelectrolyte solutions," Langmuir, vol. 15, no. 12, pp. 4069-4075, 1999.

[17] J. M'Halla and S. Boughammoura, "Translational dielectric friction and mobility of ellipsoidal polyions," Journal of Molecular Liquids, vol. 157, no. 2-3, pp. 89-101, 2010.

[18] J. M'Halla, S. Boughammoura, and A. Ghazouani, "Sharp decrease of the dielectric friction on polyions during conformation transition from pearl-chain to coiled shapes," in Proceedings of the 32iéme International Conference on Solution Chemistry (ICSI '11), la Grande Motte, France, 2011.

[19] R. Zwanzig, "Dielectric friction on a moving ion," The Journal of Chemical Physics, vol. 38, no. 7, pp. 1603-1605, 1963.

[20] J. B. Hubbard and J. F. Douglas, "Hydrodynamic friction of arbitrarily shaped Brownian particles," Physical Review E, vol. 47, no. 5, pp. R2983-R2986, 1993.

[21] D. C. Henry, "The cataphoresis of suspended particules. Part I. The equation of cataphoresis," Proceedings of the Royal Society A, vol. 133, no. 821, pp. 106-129, 1931.

[22] O. Bernard, P. Turq, and L. Blum, "Electrophoretic mobility in polyelectrolyte solutions," The Journal of Physical Chemistry, vol. 95, no. 23, pp. 9508-9513, 1991.

[23] J. Hubbard and L. Onsager, "Dielectric dispersion and dielectric friction in electrolyte solutions. I," The Journal of Chemical Physics, vol. 67, no. 11, pp. 4850-4857, 1977.
[24] P. G. Wolynes, "Molecular theory of solvated ion dynamics," The Journal of Chemical Physics, vol. 68, no. 2, pp. 473-483, 1978.

[25] H. S. Frank, "Solvent models and the interpretation of ionization and solvation phenomena," in Chemical Physic of Ionic Solutions, B. E. Conway and R. G. Barradas, Eds., pp. 53-66, John Wiley \& Sons, New York, NY, USA, 1966. 

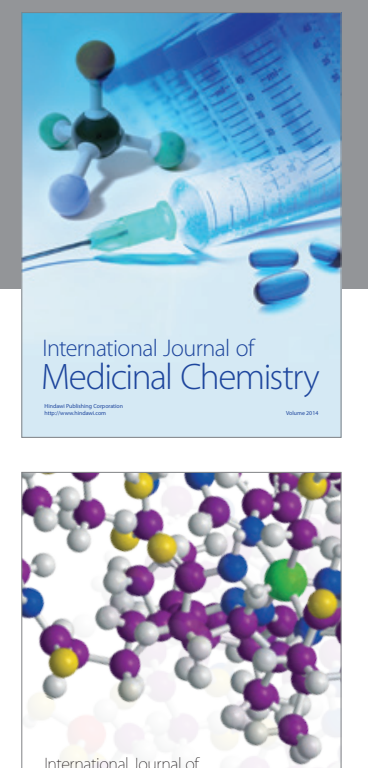

\section{Carbohydrate} Chemistry

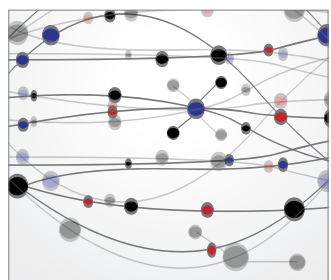

The Scientific World Journal
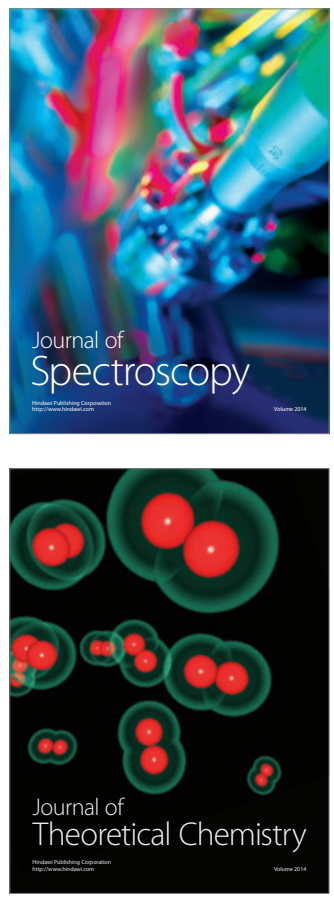
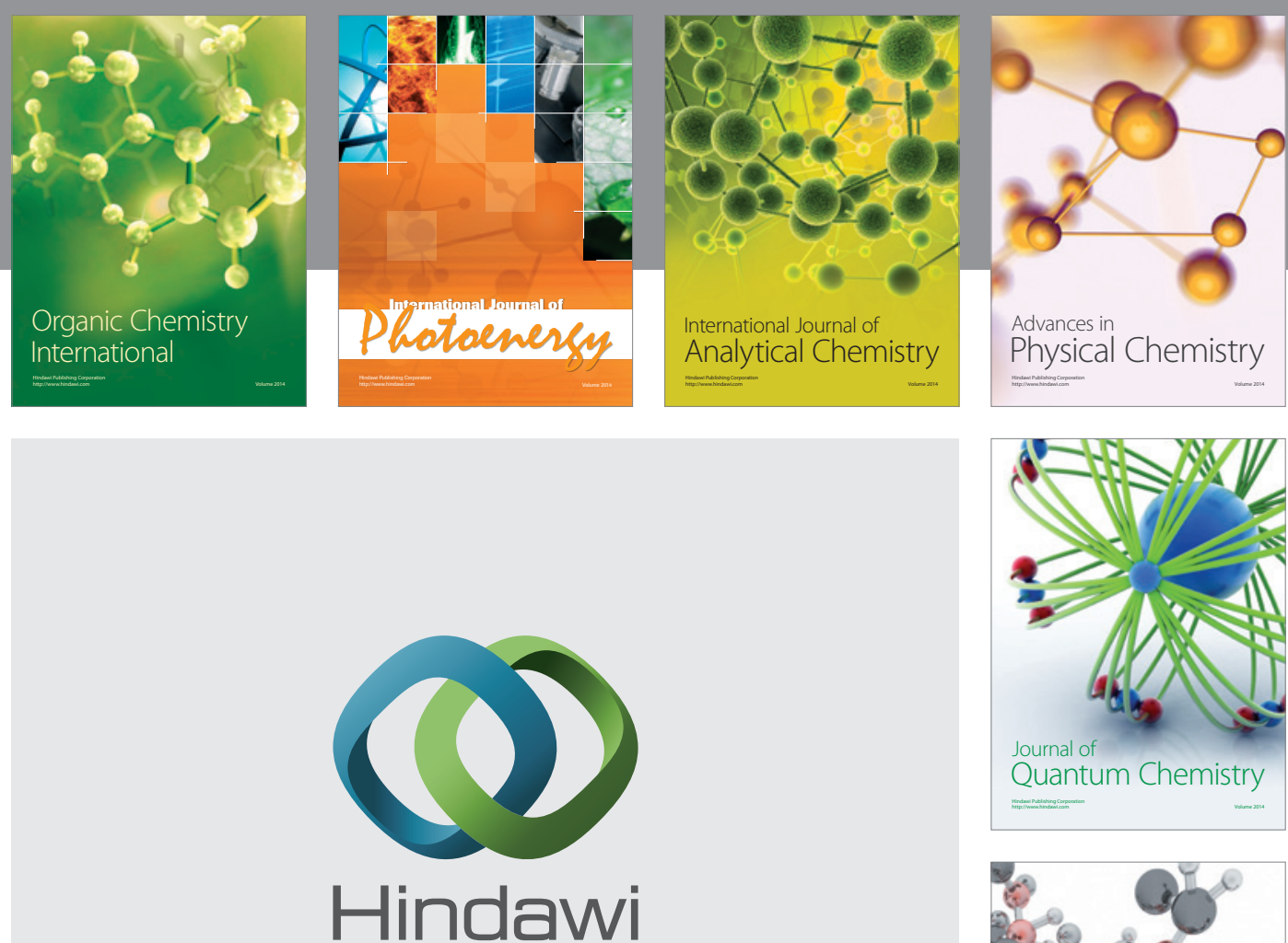

Submit your manuscripts at

http://www.hindawi.com

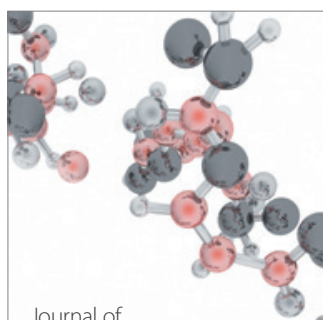

Analytical Methods

in Chemistry

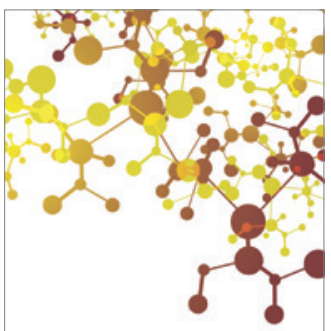

Journal of

Applied Chemistry

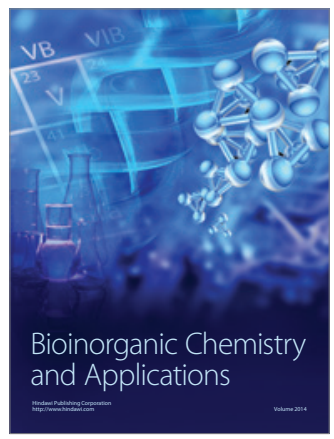

Inorganic Chemistry
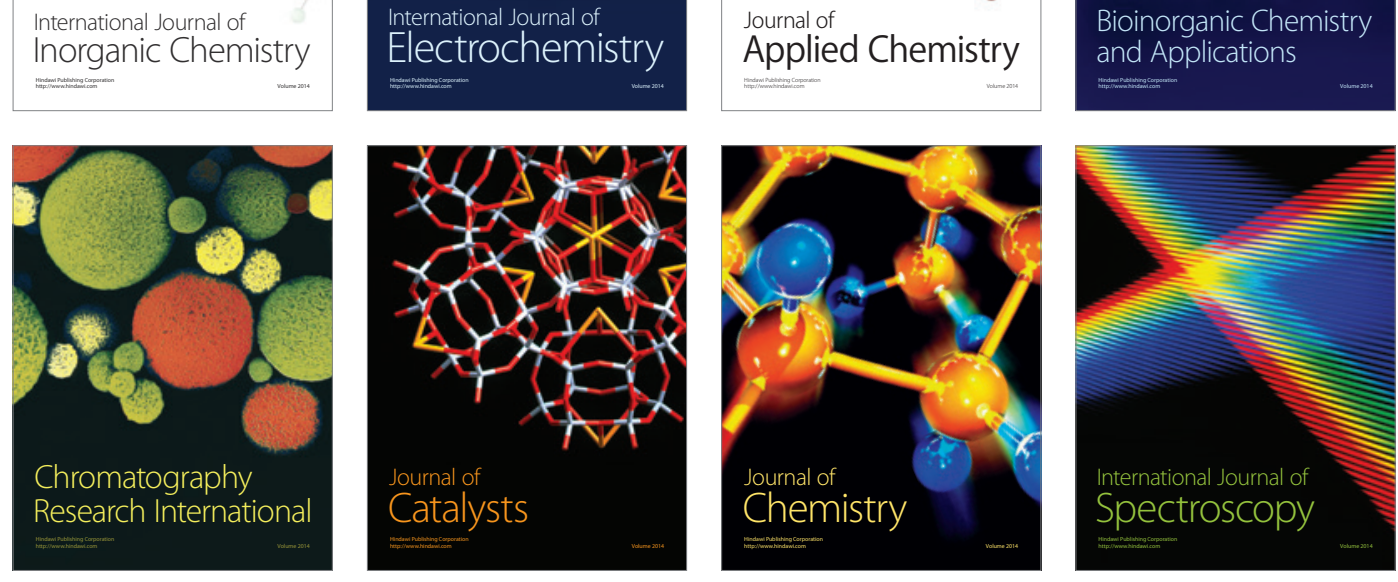